Article

\title{
Increased Oxygen Extraction by Pulmonary Rehabilitation Improves Exercise Tolerance and Ventilatory Efficiency in Advanced Chronic Obstructive Pulmonary Disease
}

\author{
Akito Miyazaki ${ }^{1}$, Keisuke Miki ${ }^{1, *}$ D, Ryoji Maekura ${ }^{2}$, Kazuyuki Tsujino ${ }^{1}$, Hisako Hashimoto ${ }^{1}$, Mari Miki $^{3}$, \\ Hiromi Yanagi ${ }^{4}$, Taro Koba ${ }^{1}$, Takuro Nii ${ }^{1}$, Takanori Matsuki ${ }^{1}$ and Hiroshi Kida ${ }^{1}$ \\ 1 Department of Respiratory Medicine, National Hospital Organization Osaka Toneyama Medical Center, \\ Toyonaka 560-8552, Japan; miyazaki.akito.qk@mail.hosp.go.jp (A.M.); \\ tsujino.kazuyuki.bh@mail.hosp.go.jp (K.T.); hashimoto.hisako.cz@mail.hosp.go.jp (H.H.); \\ koba.taro.sj@mail.hosp.go.jp (T.K.); nii.takuro.kw@mail.hosp.go.jp (T.N.); \\ matsuki.takanori.qn@mail.hosp.go.jp (T.M.); kida.hiroshi.sv@mail.hosp.go.jp (H.K.) \\ 2 Graduate School of Health Care Sciences, Jikei Institute, Osaka 532-0003, Japan; mae.ryoji@gmail.com \\ 3 Department of Internal Medicine, Tokushima Prefecture Naruto Hospital, Naruto 772-8503, Japan; \\ mmaryys@gmail.com \\ 4 Department of Clinical Examination, National Hospital Organization Osaka Toneyama Medical Center, \\ Toyonaka 560-8552, Japan; yanagi.hiromi.ex@mail.hosp.go.jp \\ * Correspondence: miki.keisuke.pu@mail.hosp.go.jp; Tel.: +81-6-6853-2001
}

Citation: Miyazaki, A.; Miki, K.; Maekura, R.; Tsujino, K.; Hashimoto, H.; Miki, M.; Yanagi, H.; Koba, T.; Nii, T.; Matsuki, T.; et al. Increased Oxygen Extraction by Pulmonary Rehabilitation Improves Exercise Tolerance and Ventilatory Efficiency in Advanced Chronic Obstructive Pulmonary Disease. J. Clin. Med. 2022, 11, 963. https://doi.org/ $10.3390 /$ jcm 11040963

Academic Editor: Gaia Cattadori

Received: 28 December 2021 Accepted: 10 February 2022 Published: 12 February 2022

Publisher's Note: MDPI stays neutral with regard to jurisdictional claims in published maps and institutional affiliations.

Copyright: (C) 2022 by the authors. Licensee MDPI, Basel, Switzerland. This article is an open access article distributed under the terms and conditions of the Creative Commons Attribution (CC BY) license (https:// creativecommons.org/licenses/by/ $4.0 /)$

\begin{abstract}
Background: In cardiopulmonary exercise testing (CPET), oxygen uptake $\left(V^{\prime} \mathrm{O} 2\right)$ is calculated using the product of minute ventilation $\left(V_{\mathrm{E}}^{\prime}\right)$ and the difference between inspiratory and expiratory $\mathrm{O}_{2}$ concentrations $\left(\Delta \mathrm{FO}_{2}\right)$. However, little is known about the response of $\Delta \mathrm{FO}_{2}$ to pulmonary rehabilitation (PR). The aim of the present study was (1) to investigate whether PR increases peak $V_{\mathrm{O} 2}^{\prime}$, based on whether $\Delta \mathrm{FO}_{2}$ or $V_{\mathrm{E}}^{\prime}$ at peak exercise increase after $\mathrm{PR}$, and (2) to investigate whether an improvement in $\Delta \mathrm{FO}_{2}$ correlates with an improvement in ventilatory efficiency. Methods: A total of 38 patients with severe and very severe COPD, whose PR responses were evaluated by $\mathrm{CPET}$, were retrospectively analyzed. Results: After PR, peak $V_{\mathrm{O} 2}^{\prime}$ was increased in 14 patients. The difference in $\Delta \mathrm{FO}_{2}$ at peak exercise following PR correlated with the difference in peak $V^{\prime} \mathrm{O} 2$ $(\mathrm{r}=0.4884, p=0.0019)$, the difference in $V_{\mathrm{E}}^{\prime} / V^{\prime} \mathrm{CO}$-nadir $(\mathrm{r}=-0.7057, p<0.0001)$, and the difference in $V^{\prime}{ }_{\mathrm{E}}-V^{\prime} \mathrm{CO} 2$ slope $(\mathrm{r}=-0.4578, p=0.0039)$, but it did not correlate with the difference in peak $V^{\prime} \mathrm{E}$. Conclusions: The increased $\mathrm{O}_{2}$ extraction following PR correlated with improved exercise tolerance and ventilatory efficiency. In advanced COPD patients, a new strategy for improving $\mathrm{O}_{2}$ extraction ability might be effective in those in whom ventilatory ability can be only minimally increased.
\end{abstract}

Keywords: dyspnea; exercise training; oxygen uptake; ventilation

\section{Introduction}

Since 2018, chronic obstructive pulmonary disease (COPD) has become the world's third leading cause of death [1]. The most frequent and intractable problem in patients with COPD is exercise intolerance due to wasted ventilation [2-5], which leads to poor disease prognosis [6]. Although several measures have been attempted to improve exercise tolerance, they remain insufficient.

It is widely accepted that pulmonary rehabilitation (PR), serving as an effective nonpharmacological intervention for COPD, improves endurance, quality of life and exertional dyspnea [7,8]. Consequently, since the survival of patients with COPD is also improved, PR is considered a very useful treatment for COPD patients $[9,10]$. The response to PR, however, varies significantly between patients, and PR including exercise training does not necessarily increase peak oxygen uptake $\left(V^{\prime}{ }_{\mathrm{O} 2}\right)$, especially in patients with advanced COPD $[11,12]$. Physical exercise requires gas exchange of both oxygen $\left(\mathrm{O}_{2}\right)$ and carbon 
dioxide $\left(\mathrm{CO}_{2}\right)$ and involves the interaction of pulmonary, cardiovascular and muscle crosstalk in the body $[6,13]$. Hence, $V^{\prime}{ }_{\mathrm{O} 2}$, as determined by cardiopulmonary exercise testing $(\mathrm{CPET})$, is calculated using the product of minute ventilation $\left(V_{\mathrm{E}}^{\prime}\right)$, as a measure of ventilatory ability, and the difference between inspired and expired $\mathrm{O}_{2}$ concentrations $\left(\Delta \mathrm{FO}_{2}\right)$, as a measure of $\mathrm{O}_{2}$ extraction ability. Therefore, peak $V_{\mathrm{O} 2}^{\prime}$ is one of the variables used to characterize total exercise ability and might be informative for assessing the efficacy of PR. In addition, reduced peak $V_{\mathrm{O} 2}^{\prime}$ has been proven to predict a poor prognosis in patients with COPD [14,15], and effective evaluation of peak $V^{\prime}{ }_{\mathrm{O} 2}$ might serve as a guide for decision-making to confirm the pathophysiological condition and choose suitable treatment for COPD.

In several chronic cardiopulmonary diseases, ventilatory inefficiency, indicated by a high $V_{\mathrm{E}}^{\prime}$ versus volume of exhaled carbon dioxide $\left(V^{\prime} \mathrm{CO}_{2}\right)$ relationship during exercise, i.e., a high $V_{\mathrm{E}}^{\prime}-V_{\mathrm{CO} 2}^{\prime}$ slope, is used as a prognostic marker and is commonly associated with a low arterial carbon dioxide partial pressure $\left(\mathrm{PaCO}_{2}\right)$ during exercise [16-18]. Recently, we reported that a high $V^{\prime}{ }_{\mathrm{E}}-V^{\prime} \mathrm{CO}_{2}$ slope was more strongly associated with a low $\Delta \mathrm{FO}_{2}$ at peak exercise, as a gas exchange parameter related to $\mathrm{O}_{2}$, as compared to $\mathrm{CO}_{2}$-related variables, such as $\mathrm{PaCO}_{2}$ and partial pressure of end-tidal $\mathrm{CO}_{2}\left(\mathrm{PetCO}_{2}\right)$, in patients with COPD [19]. Furthermore, we reported that the dependence of reduced peak $V^{\prime}{ }_{\mathrm{O} 2}$ on $\Delta \mathrm{FO}_{2}$ becomes relatively high with the progression of COPD, due to a decrease in its dependence on ventilatory ability at peak exercise, and that increasing $\Delta \mathrm{FO}_{2}$ might be an attractive approach for improving exercise tolerance and ventilatory efficiency, especially in advanced COPD patients [19].

The aim of the present study was: (1) to investigate whether PR increases exercise tolerance, evaluated by assessment of peak $V_{\mathrm{O} 2}^{\prime}$ during incremental CPET, and to assess the effect of PR based on the variables that are more significantly associated with improvement in peak $V^{\prime}{ }_{\mathrm{O} 2}$, i.e., $\Delta \mathrm{FO}_{2}$ or $V_{\mathrm{E}}^{\prime}$ at peak exercise and (2) to investigate whether an improvement in $\Delta \mathrm{FO}_{2}$ is predictive of an improvement in ventilatory efficiency, indicated by the $V_{\mathrm{E}}^{\prime}-V_{\mathrm{CO} 2}^{\prime}$ slope and the lowest value of $V_{\mathrm{E}}^{\prime} / V_{\mathrm{CO} 2}^{\prime}$ during exercise in patients with severe and very severe COPD.

\section{Materials and Methods}

\subsection{Study Design}

This retrospective study was conducted by analyzing data obtained from severe and very severe COPD patients who underwent PR while in hospital and were evaluated using CPET before and after PR at the National Hospital Organization (NHO) Osaka Toneyama Medical Center from April 2000 to July 2021. This study included data from previous ethically-approved studies performed as screening for studies on COPD at our institution. Thus, a total of 38 patients diagnosed and classified as stage III or IV COPD according to the Global Initiative for Chronic Obstructive Lung Disease (GOLD) criteria [20] were analyzed. Patients were excluded if they met the following criteria: (1) if they had a diagnosis of bronchial asthma, active infection, or severe heart disease, (2) had a history of lung resection, (3) if their drug regimens were changed within 4 weeks before PR, (4) if new treatment was added during PR, and (5) if their conditions were unstable due to other reasons. This study was performed in accordance with the Declaration of Helsinki and the institutional review board of the NHO Osaka Toneyama Medical Center approved this study (approval number: TNH-A-2021022). Written, informed consent was obtained from each patient before the first CPET evaluation.

\subsection{Pulmonary Rehabilitation (PR)}

Education and instruction, physical therapy, exercise training and occupational therapy were included in the PR program and performed in hospital. The patients were instructed to use educational material to increase their knowledge of the disease and to improve their management of it. They underwent three sets of exercise training per day with electromechanically braked cycle ergometers, from three to five days a week for one to 
two months (20 days), with high-intensity targets as previously described [21]. The initial exercise level was set for $6 \mathrm{~min}$ a set at the work rate equivalent to $60 \%$ of the baseline peak $V_{\mathrm{O} 2}^{\prime}$ before PR. If they could tolerate the exercise, the exercise duration was first increased to $10 \mathrm{~min}$ a set, following which the work rate was increased by $5 \mathrm{~W}$ and if possible, increased to the work rate equivalent to $80 \%$ of the baseline peak $V^{\prime} \mathrm{O} 2$. If the patients could not tolerate the exercise, their exercise levels were reduced to the previous setting.

\subsection{Pulmonary Function Tests (PFTs)}

Post-bronchodilator spirometry (CHESTAC 8800; CHEST M.I. Inc., Tokyo, Japan) was performed according to the recommendations of the American Thoracic Society [22]. PFTs were performed within 1 week before and after PR.

\subsection{Six-Minute Walk Test}

The six-minute walk distance (6-MWD) was measured as described previously [23]. The patients were instructed to walk at their own pace but to cover as much ground as possible in $6 \mathrm{~min}$ without encouragement.

\subsection{Cardiopulmonary Exercise Testing (CPET)}

Symptom-limited incremental exercise tests were performed using an electrically braked cycle ergometer (CV-1000SS; Lode, Groningen, The Netherlands) and a CPET system (Aero monitor AE310S; Minato Medical Science Co., Ltd., Osaka, Japan) before and after PR with the same protocol, i.e., the two-minute stage, 10-watt step protocol. Before CPET, patients were instructed to perform to their maximal effort but were advised that the exercise could be stopped at any time. During exercise, CPET was performed without encouragement until the subject was exhausted in order to achieve reliable data. All patients were instructed to maintain a speed of approximately $60 \mathrm{rpm}$ on the cycle ergometer by observing the rpm meter. Resting measurements before exercise were obtained during the steady-state period after at least $3 \mathrm{~min}$ of rest after preparation for CPET. Ventilatory values were measured on a breath-by-breath basis using a face mask and are shown as 30-s averages at rest, at two-minute intervals during exercise and at the end of exercise. Severity of dyspnea (10-point modified Borg category-ratio scale) was evaluated at rest, during the last $15 \mathrm{~s}$ of each exercise stage and at the end of exercise, and all patients were asked which symptoms (exertional dyspnea, leg discomfort, or others) caused them to stop the exercise. The $V_{\mathrm{E}}^{\prime}-V^{\prime} \mathrm{CO} 2$ slope was calculated by linear regression, excluding the nonlinear part of the data after the onset of the respiratory compensation point. If the respiratory compensation point could not be determined, the $V^{\prime}{ }_{\mathrm{E}}^{-} V^{\prime} \mathrm{CO} 2$ slope was calculated from the data from the start to the end of the exercise. The $V^{\prime}{ }_{\mathrm{E}}-V^{\prime}{ }_{\mathrm{CO} 2}$ nadir was defined as the lowest value of the ratio between $V_{\mathrm{E}}^{\prime}$ and $V^{\prime} \mathrm{CO}_{2}$ during exercise. The $V_{\mathrm{E}}^{\prime}-V^{\prime} \mathrm{CO}_{2}$ intercept was defined as the nonzero point on the $Y$-axis, i.e., $V_{\mathrm{E}}^{\prime}$ [19]. The physiological dead space to tidal volume ratio $\left(V_{\mathrm{D}} / V_{\mathrm{T}}\right)$ was estimated based on Enghoff's modification of Bohr's equation [17], using the non-invasive parameter of $\mathrm{PetCO}_{2}$ as an approximation of $\mathrm{PaCO}_{2} \cdot V_{\mathrm{D}}$-intercept was estimated as $V_{\mathrm{E}}^{\prime}-V_{\mathrm{CO} 2}^{\prime}$ intercept $/ f_{\mathrm{R}}-V_{\mathrm{CO} 2}^{\prime}$ intercept during exercise, and the $f_{\mathrm{R}}-V^{\prime} \mathrm{CO} 2$ intercept was defined as the nonzero point on the $Y$-axis, i.e., $f_{\mathrm{R}}[24]$. The time-slope was calculated as the ratio of exercise time until exhaustion to $\Delta V^{\prime} \mathrm{O} 2$ (peak minus resting $V^{\prime}{ }^{\prime}$ ) obtained during CPET [12]. The predicted maximal voluntary ventilation $(\mathrm{MVV})$ was calculated as $\mathrm{FEV}_{1} \times 35$. The predicted maximum heart rate was calculated as 220-age in years [13]. The percent predicted peak $V^{\prime} \mathrm{O} 2$ was calculated using the equations of Itoh et al. [13]. Isotime was defined as the time the shortest test ended. An inflection point of $V_{\mathrm{T}}$ during exercise was determined for each subject using the 30-s averaged data [25]. The anaerobic threshold was identified using the V-slope method [13]. 


\subsection{Statistical Analysis}

The data are expressed as means \pm standard deviation (SD). First, all patients were divided into two groups based on whether or not peak $V_{\mathrm{O} 2}^{\prime}$ increased after PR. Based on the results of variables with significant differences between the peak $V_{\mathrm{O} 2}^{\prime}$ increase group (peak $V_{\mathrm{O} 2}^{\prime}$ before $\mathrm{PR}<$ peak $V_{\mathrm{O} 2}^{\prime}$ after $\mathrm{PR}$ ) and the non-increase group (peak $V_{\mathrm{O} 2}^{\prime}$ before $\mathrm{PR} \geq$ peak $V_{\mathrm{O} 2}^{\prime}$ after $\mathrm{PR}$ ), and since $V_{\mathrm{O} 2}^{\prime}$ is calculated using the product of $V_{\mathrm{E}}^{\prime}$ and $\Delta \mathrm{FO}_{2}$, all patients were then divided into two groups based on whether $V_{\mathrm{E}}^{\prime}$ or $\Delta \mathrm{FO}_{2}$ at peak exercise had increased after PR. That is, the increase group was defined as $V^{\prime} \mathrm{E}$ or $\Delta \mathrm{FO}_{2}$ before PR was less than that after PR, and the non-increase group was defined as $V_{\mathrm{E}}^{\prime}$ or $\Delta \mathrm{FO}_{2}$ before PR was equal to or greater than that after PR. Fisher's exact test was used to compare baseline characteristics before PR between the two groups and evaluate the reasons for stopping exercise before and after PR. Mann-Whitney's U test was used to compare baseline characteristics before PR and compare the mean differences before and after PR between the two groups. The Wilcoxon signed-rank test was used to compare the results after PR with the results before PR within each group. Univariate analysis using Spearman's rank correlation coefficient as a non-parametric test was used to evaluate the correlations of $\Delta \mathrm{FO}_{2}$ at peak exercise with the other clinical variables. A $p$ value $<0.05$ was considered to indicate significance (JMP software, version 11, SAS Institute Inc., Cary, NC, USA).

\section{Results}

A total of 38 patients with severe to very severe airway obstruction according to the GOLD stages were evaluated before and after PR (Table 1). All patients were exsmokers. Before PR, all patients performed incremental CPET (mean exercise time $7.0 \mathrm{~min}$, maximum value $12.9 \mathrm{~min}$ and minimum value $2.8 \mathrm{~min}$ ), and their mean peak $V^{\prime}{ }_{\mathrm{O} 2}$ was $13.3 \mathrm{~mL} \cdot \mathrm{min}^{-1} \cdot \mathrm{kg}^{-1}$, suggesting obvious exercise intolerance.

Table 1. Patient baseline characteristics $(n=38)$.

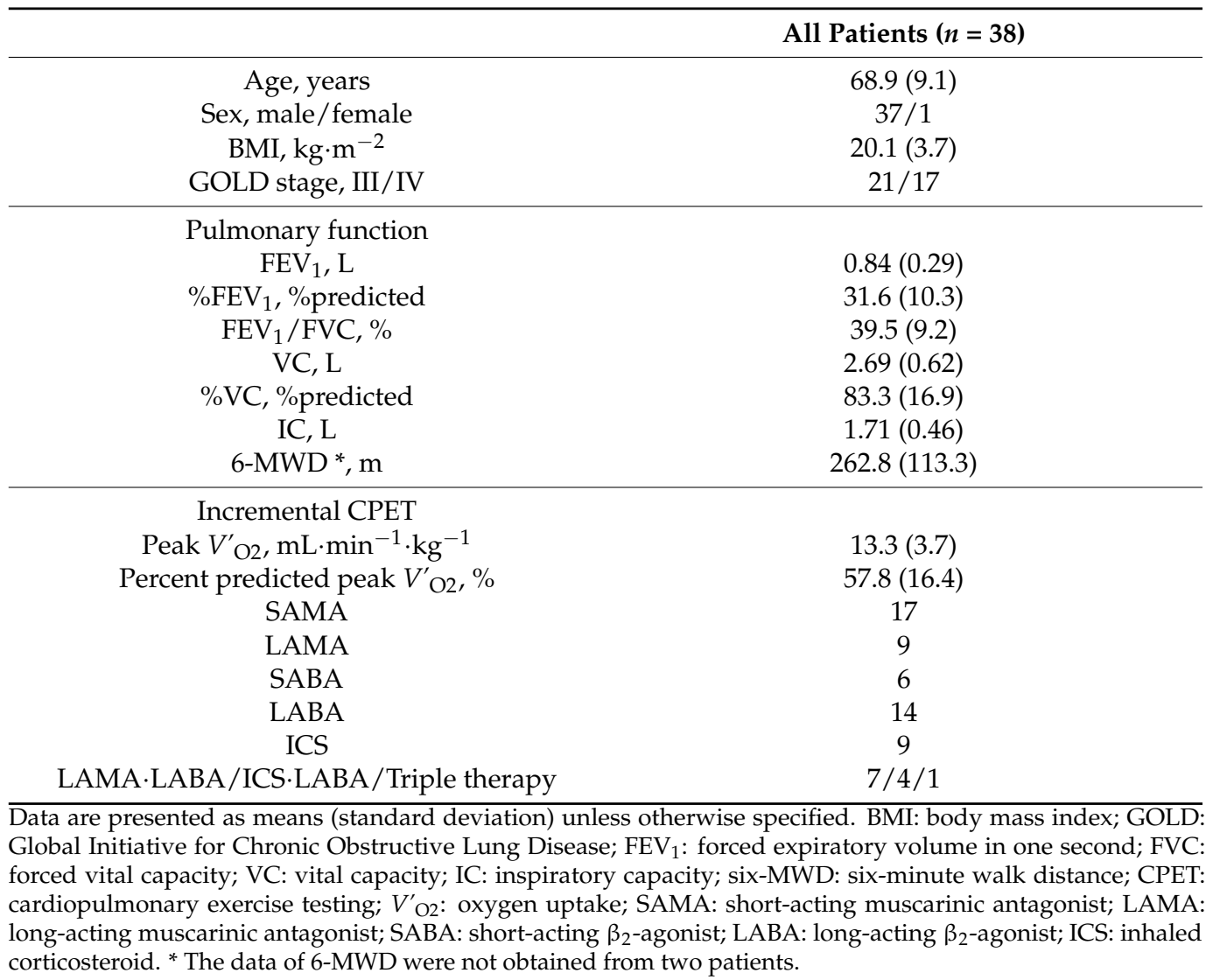


Although the difference following PR in six-MWD, which was evaluated as endurance, was a positive value in $81 \%$ of the patients, the difference following PR in peak $V^{\prime}{ }^{\prime} 2$ was positive in only $14(37 \%)$ patients (Table 2$)$. In all patients, the mean difference following PR in peak $V^{\prime}{ }^{\prime} 2$ was not increased (mean difference from pre-PR: $-0.02 \mathrm{~mL} \cdot \mathrm{min}^{-1} \cdot \mathrm{kg}^{-1}$ ). Of these 14 patients, $\triangle \mathrm{FO}_{2}$ at peak exercise during CPET after PR was increased in 11 patients $(79 \%)$, while in the remaining three patients, $V_{E}^{\prime}$ increased despite a decrease in $\Delta \mathrm{FO}_{2}$ at peak exercise after PR. In the peak $V^{\prime}{ }_{2}$ increase group, of the CPET variables obtained, the changes in the $\Delta \mathrm{FO}_{2}$ and $V_{\mathrm{E}}^{\prime}$ at peak exercise after PR were significantly higher than in the peak $V_{\mathrm{O} 2}^{\prime}$ non-increase group among CPET variables obtained (Table 2).

Table 2. Changes in cardiopulmonary variables after pulmonary rehabilitation in the peak $V^{\prime} \mathrm{O} 2$ increase and peak $V^{\prime} \mathrm{O} 2$ non-increase groups $(n=38)$.

\begin{tabular}{|c|c|c|c|c|c|}
\hline & \multicolumn{2}{|c|}{$\begin{array}{l}\text { Peak } V^{\prime}{ }^{\mathrm{O} 2} \text { Inc. Group } \\
(n=14)\end{array}$} & \multicolumn{2}{|c|}{$\begin{array}{l}\text { Peak } V_{\mathrm{O} 2}^{\prime} \text { Non-Inc. Group } \\
\qquad(n=24)\end{array}$} & \multirow[t]{2}{*}{$\begin{array}{l}p \text {-Value (Between } \\
\text { the Two Groups) }\end{array}$} \\
\hline & Pre-PR & Difference & Pre-PR & Difference & \\
\hline \multicolumn{6}{|l|}{ Pulmonary function } \\
\hline $\mathrm{FEV}_{1}, \mathrm{~L}$ & $0.77(0.26)$ & $+0.16(0.19) *$ & $0.87(0.30)$ & $+0.02(0.13)$ & 0.0514 \\
\hline $\mathrm{IC}, \mathrm{L}$ & $1.58(0.42)$ & $+0.16(0.34)$ & $1.78(0.47)$ & $-0.10(0.33)$ & 0.0735 \\
\hline 6-MWD ${ }^{\dagger}, \mathrm{m}$ & $292.3(104.6)$ & $+43.2(53.6) * *$ & $243.2(117.0)$ & $+56.8(65.8) * * *$ & 0.6373 \\
\hline \multicolumn{6}{|l|}{ Incremental CPET at peak exercise } \\
\hline Dyspnea, Borg scale & $6.6(2.5)$ & $-1.1(2.0)$ & $6.3(2.6)$ & $-1.3(2.2) * *$ & 0.8299 \\
\hline Work rate, watts & $39(9)$ & $+9(8) * *$ & $39(14)$ & $+2(10)$ & 0.0183 \\
\hline$V^{\prime} \mathrm{O} 2$ at anaerobic threshold ${ }^{+\dagger}, \mathrm{mL} \cdot \mathrm{min}^{-1}$ & $521.5(152.6)$ & $+33.6(52.6)$ & $547.4(80.0)$ & $-31.4(51.5) *$ & 0.0045 \\
\hline $\mathrm{R}$ & $1.04(0.08)$ & $+0.03(0.08)$ & $1.03(0.11)$ & $-0.01(0.05)$ & 0.0863 \\
\hline$V_{\mathrm{E}}^{\prime}, \mathrm{L} \cdot \mathrm{min}^{-1}$ & $29.3(7.4)$ & $+4.0(7.3) * *$ & $29.8(6.6)$ & $-1.2(2.5) *$ & 0.0008 \\
\hline$V_{\mathrm{T}}, \mathrm{mL}$ & $986(182)$ & $+133(154)^{* * *}$ & $1025(258)$ & +15 (153) & 0.0062 \\
\hline$f_{\mathrm{R}}$, breaths $\cdot \min ^{-1}$ & $31(8)$ & $-1(6)$ & $30(5)$ & $-1(6)$ & 0.9516 \\
\hline $\mathrm{T}_{\mathrm{i}} / \mathrm{T}_{\text {tot }}$ & $0.37(0.04)$ & $+0.01(0.04)$ & $0.36(0.07)$ & $+0.02(0.05)$ & 0.7038 \\
\hline$V_{\mathrm{D}} / V_{\mathrm{T}}$ & $0.37(0.08)$ & $-0.02(0.04)$ & $0.35(0.06)$ & $+0.02(0.05) *$ & 0.0185 \\
\hline HR, beats $\cdot \min ^{-1}$ & $116(18)$ & $+6(10)$ & $119(20)$ & $+1(16)$ & 0.1112 \\
\hline $\mathrm{O}_{2}$ pulse, $\mathrm{mL} \cdot$ beats $^{-1}$ & $6.7(1.2)$ & $+0.4(0.7) *$ & $6.0(1.5)$ & $-0.5(0.5) * * * *$ & 0.0001 \\
\hline $\mathrm{SpO}_{2}, \%$ & $90.4(4.6)$ & $0(2.5)$ & $89.5(5.5)$ & $-0.6(3.2)$ & 0.4565 \\
\hline $\mathrm{PetCO}_{2}, \mathrm{mmHg}$ & $37.8(6.7)$ & $+0.4(3.2)$ & $37.3(4.9)$ & $-1.3(3.5)$ & 0.1463 \\
\hline$\Delta \mathrm{FO}_{2}, \%$ & $2.79(0.50)$ & $+0.15(0.33)$ & $2.91(0.42)$ & $-0.20(0.30) * *$ & 0.0037 \\
\hline$V_{\mathrm{D}}$-intercept $/ V_{\mathrm{T}}$ & $0.46(0.20)$ & $-0.01(0.41)$ & $0.40(0.25)$ & $+0.01(0.23)$ & 0.5701 \\
\hline$V_{\mathrm{E}}^{\prime} / V_{\mathrm{CO} 2}^{\prime}$ & $43.8(7.8)$ & $-1.1(4.2)$ & $42.5(7.6)$ & $+2.6(4.2) * *$ & 0.0168 \\
\hline$V_{\mathrm{E}}^{\prime} / V_{\mathrm{CO} 2}^{\prime}$-nadir & $43.3(8.0)$ & $-1.4(3.1)$ & $42.3(7.6)$ & $+2.4(4.2) * *$ & 0.0062 \\
\hline$V_{\mathrm{E}}^{\prime}-V_{\mathrm{CO} 2}^{\prime}$-slope & $31.7(7.2)$ & $+1.3(4.0)$ & $34.3(10.2)$ & $+3.3(4.7)^{* *}$ & 0.3884 \\
\hline
\end{tabular}

Data are presented as means (standard deviation). $V_{\mathrm{O} 2}^{\prime}$ : oxygen uptake; inc.: increase; PR: pulmonary rehabilitation; $\mathrm{FEV}_{1}$ : forced expiratory volume in one second; IC: inspiratory capacity; 6-MWD: six-minute walk distance; CPET: cardiopulmonary exercise testing; R: gas exchange ratio; $V_{\mathrm{E}}^{\prime}$ : minute ventilation; $V_{\mathrm{T}}$ : tidal volume $f_{\mathrm{R}}$ : breathing frequency; $\mathrm{T}_{\mathrm{i}} / \mathrm{T}_{\text {tot }}$ : inspiratory duty cycle; $V_{\mathrm{D}} / V_{\mathrm{T}}$ : physiological dead space/tidal volume ratio; $\mathrm{HR}$ : heart rate; $\mathrm{SpO}_{2}$ : percutaneous oxygen saturation; $\mathrm{PetCO}_{2}$ : partial pressure of end-tidal carbon dioxide; $\Delta \mathrm{FO}_{2}$ : difference between inspiratory and expiratory $\mathrm{O}_{2}$ concentrations; $V_{\mathrm{D}}$-intercept: $V_{\mathrm{D}}$ calculated as $V^{\prime}{ }_{\mathrm{E}}$-axis intercept $/ f_{\mathrm{R}}$-axis intercept, obtained from $V_{\mathrm{E}}^{\prime} \mathrm{Vs} . V_{\mathrm{CO} 2}^{\prime}$ and $f_{\mathrm{R}} \mathrm{Vs}$. $V_{\mathrm{CO} 2}^{\prime}$ relationships (see the Methods section for details); $V_{\mathrm{CO} 2}^{\prime}$ : carbon dioxide output; $V_{\mathrm{E}}^{\prime} / V^{\prime} \mathrm{CO}_{2}$-nadir: lowest value of the ratio between $V_{\mathrm{E}}^{\prime}$ and $V_{\mathrm{CO} 2}^{\prime}$ during exercise (see the Methods section for details); $V_{\mathrm{E}}^{\prime}-V^{\prime} \mathrm{CO}_{2}$-slope: the slope was determined by linear regression analysis of $V_{\mathrm{E}}^{\prime}$ to $V_{\mathrm{CO} 2}^{\prime}$ relationship (see the Methods for details). ${ }^{*} p<0.05,{ }^{* *} p<0.01,{ }^{* * *} p<0.001,{ }^{* * *} p<0.0001$ : compared with pre-PR values (within-group difference). ${ }^{+}$The data of six-MWD from 2 patients were not obtained ${ }^{+\dagger}$ : 27 patients (peak $V_{\mathrm{O} 2}^{\prime}$ inc. group, $n=11$; peak $V_{\mathrm{O} 2}^{\prime}$ non-increase group, $n=16$ ), whose anaerobic thresholds were obtained before and after pulmonary rehabilitation were analyzed.

Therefore, we investigated the effects of $\mathrm{PR}$ on $\mathrm{O}_{2}$ extraction or ventilatory ability by dividing the patient cohort into two groups based on whether or not they experienced an increase in the $\Delta \mathrm{FO}_{2}$ or $V_{\mathrm{E}}^{\prime}$ at peak exercise after PR (Tables 3-5). The groups divided according to the increase in the $\Delta \mathrm{FO}_{2}$ or $V_{\mathrm{E}}^{\prime}$ at peak exercise were well matched for age, sex and body mass index (BMI), GOLD stages and resting pulmonary function, although the number of subjects with dual or triple inhalation therapy was low in each group (Table 3). In the peak $\triangle \mathrm{FO}_{2}$ increase group, compared with the change following PR in the peak $\Delta \mathrm{FO}_{2}$ non-increase group, (1) the mean differences following PR in peak $V_{\mathrm{O} 2}^{\prime}(p=0.0136)$, $V^{\prime}{ }^{\prime} 2$ at anaerobic threshold $(p<0.0001)$, and $\mathrm{PetCO}_{2}$ at peak exercise $(p=0.0007)$ were significantly increased, and those in $V_{\mathrm{E}}^{\prime} / V^{\prime} \mathrm{CO}_{2}$-nadir $(p<0.0001)$ during exercise and 
$V^{\prime}{ }^{\prime}-V_{\mathrm{CO} 2}^{\prime}$-slope $(p=0.0413)$ were significantly improved, although $V_{\mathrm{E}}^{\prime}-V_{\mathrm{CO} 2}^{\prime}$ intercept was not changed (Table 4), (2) notwithstanding that the mean difference following PR in $V_{\mathrm{T}}$ at peak exercise was increased $(p=0.0167)$, and in the $f_{\mathrm{R}}$ at peak exercise was reduced $(p=0.0049)$, the mean difference following PR in the inspiratory duty cycle (Ti/Ttot) at peak exercise $(p=0.4788)$ and in $V_{\mathrm{T}}$ at the inflection point during exercise $(p=0.1798)$ were not changed (Table 4 and Figure 1). Then, the mean difference following PR in $V_{E}^{\prime}$ did not increase significantly $(p=0.7134)$ (Table 4$)$, and (3) the mean difference following PR in $\mathrm{O}_{2}$-pulse was not significantly changed $(p=0.2218)$ (Table 4$)$. In the peak $\Delta \mathrm{FO}_{2}$ non-increase group, (i) dyspnea at peak exercise was significantly reduced, as seen in the within-group evaluation ( $p=0.0006)$, (ii) the time-slope was significantly lower before $\operatorname{PR}(p=0.0277)$ and increased after PR compared with the peak $\Delta \mathrm{FO}_{2}$ increase group $(p=0.0302)$ (Table 4$)$. In contrast, in the peak $V_{\mathrm{E}}^{\prime}$ increase group, compared with the mean difference following PR in the peak $V_{\mathrm{E}}^{\prime}$ non-increase group, although the mean difference following PR in peak $V_{\mathrm{O} 2}^{\prime}$ was significantly increased $(p=0.0109)$, those in $V_{\mathrm{O} 2}^{\prime}$ at the anaerobic threshold $(p=0.6429)$, in $V_{\mathrm{E}}^{\prime} / V^{\prime} \mathrm{CO}_{2}$-nadir during exercise $(p=0.5685)$, in $V_{\mathrm{E}}^{\prime}-V^{\prime} \mathrm{CO}_{2}$-slope $(p=1.0000)$, in $\Delta \mathrm{FO}_{2}$ $(p=0.4558)$, and in $\operatorname{PetCO}_{2}(p=0.5200)$ at peak exercise did not change, and significant tachypnea at peak exercise $(p=0.0415)$ was seen (Table 5 and Figure 2).

Table 3. Comparison between groups stratified according to the change in peak $\Delta \mathrm{FO}_{2}$ or peak $V_{\mathrm{E}}^{\prime}$ $(n=38)$ after pulmonary rehabilitation.

\begin{tabular}{|c|c|c|c|}
\hline & Peak $\Delta \mathrm{FO}_{2}$ Inc. Group $(n=17)$ & Peak $\Delta \mathrm{FO}_{2}$ Non-Inc. Group $(n=21)$ & $p$-Value \\
\hline Age, years & $71.4(8.5)$ & $66.8(9.2)$ & 0.1301 \\
\hline Sex, male/female & $16 / 1$ & $21 / 0$ & 0.2600 \\
\hline $\mathrm{BMI}, \mathrm{kg} \cdot \mathrm{m}^{-2}$ & $19.7(3.1)$ & $20.5(4.2)$ & 0.6918 \\
\hline GOLD stage, III/IV & $9 / 8$ & $12 / 9$ & 0.7956 \\
\hline \multicolumn{4}{|l|}{ Pulmonary function } \\
\hline $\mathrm{FEV}_{1}, \mathrm{~L}$ & $0.78(0.27)$ & $0.88(0.30)$ & 0.3250 \\
\hline$\% \mathrm{FEV}_{1}, \%$ predicted & $30.6(10.2)$ & $32.4(10.5)$ & 0.6281 \\
\hline $\mathrm{FEV}_{1} / \mathrm{FVC}, \%$ & $41.0(7.0)$ & $38.3(10.6)$ & 0.3041 \\
\hline VC, $\mathrm{L}$ & $2.57(0.62)$ & $2.77(0.62)$ & 0.2838 \\
\hline$\%$ VC, \%predicted & $81.8(17.3)$ & $84.5(16.8)$ & 0.6073 \\
\hline IC, L & $1.57(0.41)$ & $1.81(0.48)$ & 0.1487 \\
\hline \multicolumn{4}{|l|}{ Incremental CPET } \\
\hline peak $V_{\mathrm{O} 2}^{\prime}, \mathrm{mL} \cdot \mathrm{min}^{-1} \cdot \mathrm{kg}^{-1}$ & $13.0(4.1)$ & $13.6(3.3)$ & 0.6073 \\
\hline \multirow[t]{2}{*}{ LAMA·LABA/ICS·LABA/Triple } & $2 / 3 / 0$ & $5 / 1 / 1$ & 0.3836 \\
\hline & Peak $V_{\mathrm{E}}^{\prime}$ inc. group $(n=20)$ & Peak $V_{\mathrm{E}}^{\prime}$ non-inc. group $(n=18)$ & $p$-Value \\
\hline Age, years & $67.5(10.0)$ & $70.4(8.0)$ & 0.3960 \\
\hline Sex, male/female & $19 / 1$ & $18 / 0$ & 0.3363 \\
\hline $\mathrm{BMI}, \mathrm{kg} \cdot \mathrm{m}^{-2}$ & $20.6(4.7)$ & $19.7(2.4)$ & 0.6295 \\
\hline GOLD stage, III/IV & $12 / 8$ & $9 / 9$ & 0.5359 \\
\hline \multicolumn{4}{|l|}{ Pulmonary function } \\
\hline $\mathrm{FEV}_{1}, \mathrm{~L}$ & $0.88(0.30)$ & $0.78(0.27)$ & 0.2856 \\
\hline$\% \mathrm{FEV}_{1}, \%$ predicted & $33.1(10.2)$ & $30.0(10.4)$ & 0.4047 \\
\hline $\mathrm{FEV}_{1} / \mathrm{FVC}, \%$ & $40.6(9.4)$ & $38.2(9.0)$ & 0.5296 \\
\hline VC, L & $2.79(0.66)$ & $2.55(0.56)$ & 0.2193 \\
\hline$\% \mathrm{VC}, \%$ & $86.5(17.5)$ & $79.7(15.8)$ & 0.1561 \\
\hline $\mathrm{IC}, \mathrm{L}$ & $1.74(0.51)$ & $1.68(0.42)$ & 0.6808 \\
\hline \multicolumn{4}{|l|}{ Incremental CPET } \\
\hline peak $V_{\mathrm{O} 2}^{\prime}, \mathrm{mL} \cdot \mathrm{min}^{-1} \cdot \mathrm{kg}^{-1}$ & $13.1(3.6)$ & $13.6(3.9)$ & 0.7366 \\
\hline LAMA·LABA/ICS·LABA/Triple & $1 / 3 / 1$ & $6 / 1 / 0$ & 0.0855 \\
\hline
\end{tabular}

Data are presented as means (standard deviation) unless otherwise specified. $\Delta \mathrm{FO}_{2}$ : difference between inspiratory and expiratory $\mathrm{O}_{2}$ concentrations; $V_{\mathrm{E}}^{\prime}$ : minute ventilation; inc.: increase; BMI: body mass index; GOLD: Global Initiative for Chronic Obstructive Lung Disease; $\mathrm{FEV}_{1}$ : forced expiratory volume in one second; FVC: forced vital capacity; VC: vital capacity; IC: inspiratory capacity; CPET: cardiopulmonary exercise testing; $V^{\prime} \mathrm{O} 2$ : oxygen uptake; LAMA: long-acting muscarinic antagonist; LABA: long-acting $\beta_{2}$-agonist; ICS: inhaled corticosteroid. 
Table 4. Changes in the results of exercise testing after pulmonary rehabilitation in the peak $\Delta \mathrm{FO}_{2}$ increase and non-increase groups.

\begin{tabular}{|c|c|c|c|c|c|}
\hline & \multicolumn{2}{|c|}{$\begin{array}{c}\text { Peak } \Delta \mathrm{FO}_{2} \text { Inc. Group } \\
(n=17)\end{array}$} & \multicolumn{2}{|c|}{$\begin{array}{l}\text { Peak } \Delta \mathrm{FO}_{2} \text { Non-Inc. Group } \\
(n=21)\end{array}$} & \multirow[t]{2}{*}{$\begin{array}{l}p \text {-Value (Between } \\
\text { the Two Groups) }\end{array}$} \\
\hline & Pre-PR & Difference & Pre-PR & Difference & \\
\hline \multicolumn{6}{|l|}{$\begin{array}{c}\text { Incremental CPET at peak } \\
\text { exercise }\end{array}$} \\
\hline Dyspnea, Borg scale & $6.3(2.9)$ & $-0.8(2.4)$ & $6.5(2.3)$ & $-1.6(1.8) * * *$ & 0.2457 \\
\hline$V_{\mathrm{O} 2}^{\prime}, \mathrm{ml} \cdot \mathrm{min}^{-1} \cdot \mathrm{kg}^{-1}$ & $13.0(4.1)$ & $+0.7(1.7)$ & $13.6(3.3)$ & $-0.6(2.2)$ & 0.0136 \\
\hline Exercise time, sec & $416(154)$ & $+58(72)^{* *}$ & $418(160)$ & $+51(113)$ & 0.8717 \\
\hline Work rate, watts & $39(12)$ & $+6(7) * *$ & $40(13)$ & $+4(12)$ & 0.5296 \\
\hline $\begin{array}{c}V_{\mathrm{O} 2}^{\prime} \text { at anaerobic threshold } \\
\mathrm{mL} \cdot \mathrm{min}^{-1}\end{array}$ & $502.3(111.8)$ & $+50.3(38.7)^{* * *}$ & $564.5(110.3)$ & $-49.1(30.0)^{* * * *}$ & $<0.0001$ \\
\hline $\mathrm{R}$ & $1.02(0.10)$ & $+0.02(0.06)$ & $1.03(0.10)$ & $+0.00(0.08)$ & 0.3104 \\
\hline$V_{\mathrm{E}}^{\prime}, \mathrm{L} \cdot \mathrm{min}^{-1}$ & $29.2(8.0)$ & $-0.1(2.7)$ & $29.9(5.9)$ & $+1.4(6.8)$ & 0.7134 \\
\hline$V_{\mathrm{T}}, \mathrm{mL}$ & $986(206)$ & $+110(153)^{* *}$ & $1031(253)$ & +16 (159) & 0.0167 \\
\hline \multirow{2}{*}{$\begin{array}{l}V_{\mathrm{T}} \text { at inflection point, } \mathrm{mL} \\
f_{\mathrm{R}}, \text { breaths } \cdot \mathrm{min}^{-1}\end{array}$} & $1040(204)$ & $+155(195) *$ & $1029(231)$ & $+76(194)$ & 0.1798 \\
\hline & $30(5)$ & $-3(4)^{* *}$ & $31(7)$ & $0(7)$ & 0.0049 \\
\hline $\mathrm{T}_{\mathrm{i}} / \mathrm{T}_{\text {tot }}$ & $0.37(0.05)$ & $0(0.04)$ & $0.36(0.07)$ & $+0.02(0.05)$ & 0.4788 \\
\hline$V_{\mathrm{D}} / V_{\mathrm{T}}$ & $0.38(0.06)$ & $-0.01(0.04)$ & $0.34(0.07)$ & $+0.02(0.05) *$ & 0.0534 \\
\hline HR, beats $\cdot \min ^{-1}$ & $113(20)$ & $+6(9) *$ & $122(18)$ & $0(17)$ & 0.2210 \\
\hline $\mathrm{O}_{2}$ pulse, $\mathrm{mL} \cdot$ beats $^{-1}$ & $5.8(1.5)$ & $-0.1(0.6)$ & $5.9(1.4)$ & $-0.3(0.8) *$ & 0.2218 \\
\hline $\mathrm{SpO}_{2}, \%$ & $89.6(5.4)$ & $-0.9(2.5)$ & $90.0(5.0)$ & $+0.0(3.3)$ & 0.3656 \\
\hline $\mathrm{PetCO}_{2}, \mathrm{mmHg}$ & $37.8(5.8)$ & $+1.4(3.0)$ & $37.2(5.5)$ & $-2.4(2.8) * * *$ & 0.0007 \\
\hline$V_{\mathrm{E}}^{\prime} / V_{\mathrm{CO} 2}^{\prime}$ & $44.4(7.1)$ & $-2.4(2.8)^{* *}$ & $418(8.0)$ & $+4.2(3.4)^{* * * *}$ & $<0.0001$ \\
\hline & $6.6(2.6)$ & $-0.5(1.6)$ & $6.0(3.4)$ & $-0.8(2.7)$ & 0.9298 \\
\hline $\begin{array}{l}V \mathrm{E}^{-V} \mathrm{CO}^{-1 n t e r c e p t}, \mathrm{~L} \cdot \mathrm{min} \\
V_{\mathrm{D}^{-} \text {-intercept }} / V_{\mathrm{T}}\end{array}$ & $0.44(0.26)$ & $+0.10(0.36)$ & $0.41(0.20)$ & $-0.07(0.24)$ & 0.4738 \\
\hline$V_{\mathrm{E}}^{\prime} / V_{\mathrm{CO} 2}^{\prime}$-nadir & $44.0(7.4)$ & $-2.1(2.6) * *$ & $41.6(7.9)$ & $+3.5(3.5) * * * *$ & $<0.0001$ \\
\hline$V_{\mathrm{E}}^{\prime}-V_{\mathrm{CO} 2}^{\prime}$-slope & $32.5(6.7)$ & $+0.8(3.9)$ & $34.0(10.9)$ & $+4.1(4.5) * * *$ & 0.0413 \\
\hline Time slope, sec $\cdot \mathrm{mL}^{-1} \cdot \mathrm{min}$ & $1.04(0.29)^{\dagger}$ & $+0.02(0.33)$ & $0.91(0.30)$ & $+0.20(0.20)^{* * *}$ & 0.0302 \\
\hline The causes to stop during CPET & Pre-PR & Post-PR & Pre-PR & Post-PR & Not evaluated \\
\hline \multirow[t]{2}{*}{ Dyspnea/leg fatigue } & $10 / 7$ & $9 / 8$ & $15 / 6$ & $11 / 10 *$ & Not evaluated \\
\hline & $\begin{array}{l}\text { are presented as } \\
\text { ncentrations at } \\
\text { nce; CPET: cardi } \\
\text { lation; } V_{\mathrm{T}} \text { : tidal v } \\
/ \text { tidal volume } \mathrm{r} \\
l \text { pressure of end } \\
\text { cept } / f_{\mathrm{R}} \text {-axis inte } \\
\text { etails); } V_{\mathrm{E}}^{\prime} / V^{\prime} \mathrm{CO} \\
\mathrm{n} \text { for details); } V \\
\text { ned during exerc } \\
\mathrm{V}^{\prime} \mathrm{O} \text { (peak minu } \\
0.001, * * * *<<0 \\
\mathrm{R} \text { values betweer } \\
\Delta \mathrm{FO} \mathrm{F}_{2} \text { inc. group } \\
\text { e and after pulm }\end{array}$ & $\begin{array}{l}\text { eans (standard de } \\
\text { ak exercise; inc.: } \\
\text { bulmonary exercis } \\
\text { ume; } f_{\mathrm{R}} \text { : breathing } \\
\text { o; HR: heart rate; } \\
\text { dal carbon dioxide; } \\
\text { ept, obtained from } \\
\text { adir: lowest value } \\
-V^{\prime} \mathrm{CO}^{-} \text {-slope: the } \\
\text { (see the Methods } \\
\text { esting } V^{\prime} \mathrm{O} \text { ) durin } \\
01 \text { : compared witl } \\
\text { he two groups. }{ }^{\ddagger} \mathrm{Tl} \\
l=12 \text {; peak } \Delta \mathrm{FO} \mathrm{O}_{2} \mathrm{n} \\
\text { ary rehabilitation }\end{array}$ & $\begin{array}{l}\text { tion). Peak } \triangle \mathrm{FO} \\
\text { rease; PR: pulm } \\
\text { esting; } V_{\mathrm{O} 2}^{\prime} \text { : oxy } \\
\text { quency; } \mathrm{T}_{\mathrm{i}} / \mathrm{T}_{\text {tot }}: \\
\text { pulse: } V_{\mathrm{O}}^{\prime} / \mathrm{HR} \text {; } \\
\mathrm{CO}: \text { carbon diox } \\
\text { vs. } V_{\mathrm{CO}}^{\prime} \text { and } f_{\mathrm{I}} \\
\text { the ratio betwee } \\
\text { pe was determir } \\
\text { tion for details); } \\
\text {-PET (see the Mr } \\
\text { re-PR values (wi } \\
\text { data of 6-MWD w } \\
\text {-increase group, } \\
\text { re analyzed. }\end{array}$ & $\begin{array}{l}\text { difference between i } \\
\text { ary rehabilitation; si } \\
\text { n uptake; R: gas exc } \\
\text { oiratory duty cycle; } \\
\mathrm{O}_{2} \text { : percutaneous o } \\
\text { output; } V_{\mathrm{D}^{-} \text {-intercept }} \\
\text { s. } V^{\prime} \mathrm{CO} 2 \text { relationship } \\
{ }^{\prime} \text { and } V^{\prime} \mathrm{CO} 2 \text { during } \\
\text { by linear regressior } \\
\text { ne slope: ratio of exe } \\
\text { ods section for deta } \\
\text { n-group difference). } \\
\text { not obtained from } t \\
\text { 15), whose anaerobi }\end{array}$ & $\begin{array}{l}\text { piratory and expiratory } \\
\text { MWD: six-minute walk } \\
\text { ange ratio; } V^{\prime}{ }_{\mathrm{E}}: \text { minute } \\
/ V_{\mathrm{T}} \text { : physiological dead } \\
\text { gen saturation; } \mathrm{PetCO}_{2} \\
V_{\mathrm{D}} \text { calculated as } V^{\prime} \mathrm{E}^{-a x i} \\
\text { (see the Methods section } \\
\text { xercise (see the Methods } \\
\text { analysis of } V_{\mathrm{E}}^{\prime} \text { to } V^{\prime} \mathrm{CO} 2 \\
\text { ise time until exhaustion } \\
\text { ). }{ }^{*} p<0.05,{ }^{* *} p<0.01 \\
p<0.05: \text { compared with } \\
\text { o patients. }{ }^{++}: 27 \text { patients } \\
\text { hresholds were obtained }\end{array}$ \\
\hline
\end{tabular}


Table 5. Changes in the results of exercise testing after pulmonary rehabilitation in the peak $V_{\mathrm{E}}^{\prime}$ increase and non-increase groups.

\begin{tabular}{|c|c|c|c|c|c|}
\hline & \multicolumn{2}{|c|}{$\begin{array}{l}\text { Peak } V_{\mathrm{E}}^{\prime} \text { Inc. Group } \\
(n=20)\end{array}$} & \multicolumn{2}{|c|}{$\begin{array}{l}\text { Peak } V_{\mathrm{E}}^{\prime} \text { Non-Inc. Group } \\
(n=18)\end{array}$} & \multirow[t]{2}{*}{$\begin{array}{l}p \text {-Value (Between } \\
\text { the Two Groups) }\end{array}$} \\
\hline & Pre-PR & Difference & Pre-PR & Difference & \\
\hline $\begin{array}{c}\text { 6-MWD }{ }^{\dagger}, \mathrm{m} \\
\text { Incremental CPET at peak } \\
\text { exercise }\end{array}$ & $268.9(102.8)$ & $+43.7(55.1)$ & $256.4(126.4)$ & $+59.5(67.0)$ & 0.5860 \\
\hline Dyspnea, Borg scale & $7.3(2.0)$ & $-1.8(2.1)^{* * *}$ & $5.5(2.8)$ & $-0.6(2.0)$ & 0.0778 \\
\hline$V_{\mathrm{O} 2}^{\prime}, \mathrm{ml} \cdot \mathrm{min}^{-1} \cdot \mathrm{kg}^{-1}$ & $13.1(3.6)$ & $+0.8(2.3)$ & $13.6(3.9)$ & $-0.9(1.4)^{* *}$ & 0.0109 \\
\hline Exercise time, sec & $422(147)$ & $+77(110)^{* *}$ & $411(167)$ & $+29(72) *$ & 0.1077 \\
\hline Work rate, watts & $41(11)$ & $+6(11)$ & $38(14)$ & $+4(8)$ & 0.8725 \\
\hline $\begin{array}{c}V_{\mathrm{O} 2}^{\prime} \text { at anaerobic threshold }{ }^{\dagger+}, \\
\mathrm{mL} \cdot \mathrm{min}^{-1}\end{array}$ & $536.0(124.6)$ & $-1.7(64.1)$ & $538.0(102.7)$ & $-9.0(58.1)$ & 0.6429 \\
\hline $\mathrm{R}$ & $1.04(0.10)$ & $+0.02(0.07)$ & $1.02(0.11)$ & $-0.01(0.06)$ & 0.3404 \\
\hline$V_{\mathrm{T}}, \mathrm{mL}$ & $1043(251)$ & $+79(160)^{*}$ & $976(209)$ & $+35(165)$ & 0.1932 \\
\hline$V_{\mathrm{T}}$ at inflection point, $\mathrm{mL}$ & $1058(224)$ & $+102(167) *$ & $1003(212)$ & $+118(231)$ & 0.9683 \\
\hline$f_{\mathrm{R}}$, breaths $\cdot \mathrm{min}^{-1}$ & $30(7)$ & $+1(5)$ & $30(6)$ & $-3(6) *$ & 0.0415 \\
\hline $\mathrm{T}_{\mathrm{i}} / \mathrm{T}_{\text {tot }}$ & $0.36(0.05)$ & $+0.01(0.04)$ & $0.36(0.07)$ & $+0.02(0.05)$ & 0.6593 \\
\hline$V_{\mathrm{D}} / V_{\mathrm{T}}$ & $0.36(0.08)$ & $+0.00(0.05)$ & $0.36(0.05)$ & $+0.00(0.05)$ & 0.9415 \\
\hline $\mathrm{HR}$, beats $\cdot \min ^{-1}$ & $122(17)$ & $+6(12)$ & $114(21)$ & $-1(16)$ & 0.4035 \\
\hline $\mathrm{O}_{2}$ pulse, $\mathrm{mL} \cdot$ beats $^{-1}$ & $5.6(1.2)$ & $+0.1(0.7)$ & $6.1(1.6)$ & $-0.5(0.6)^{* *}$ & 0.0108 \\
\hline $\mathrm{SpO}_{2}, \%$ & $90.3(4.7)$ & $+0.1(2.8)$ & $89.3(5.6)$ & $-0.9(3.1)$ & 0.1723 \\
\hline $\mathrm{PetCO}_{2}, \mathrm{mmHg}$ & $37.5(6.3)$ & $-1.0(2.8)$ & $37.4(4.7)$ & $-0.4(4.1)$ & 0.5200 \\
\hline$\Delta \mathrm{FO}_{2}, \%$ & $2.85(0.50)$ & $-0.10(0.34)$ & $2.89(0.41)$ & $-0.03(0.37)$ & 0.4558 \\
\hline$V_{\mathrm{E}}^{\prime} / V_{\mathrm{CO} 2}^{\prime}$ & $43.2(8.3)$ & $+1.8(4.9)$ & $42.8(7.1)$ & $+0.6(4.2)$ & 0.2792 \\
\hline$V_{\mathrm{E}}^{\prime}-V_{\mathrm{CO} 2}^{\prime}$-intercept, $\mathrm{L} \cdot \mathrm{min}^{-1}$ & $6.7(2.7)$ & $-0.2(2.0)$ & $5.9(3.3)$ & $-1.2(2.4) *$ & 0.1883 \\
\hline$V_{\mathrm{D}}$-intercept $/ V_{\mathrm{T}}$ & $0.44(0.20)$ & $-0.01(0.37)$ & $0.41(0.27)$ & $0.03(0.22)$ & 0.6674 \\
\hline$V_{\mathrm{E}}^{\prime} / V_{\mathrm{CO} 2}^{\prime}$-nadir & $42.9(8.3)$ & $+1.2(4.4)$ & $42.4(7.1)$ & $+0.8(4.1)$ & 0.5685 \\
\hline$V_{\mathrm{E}}^{\prime}-V_{\mathrm{CO} 2}^{\prime}$-slope & $32.8(8.5)$ & $+2.6(4.6)^{*}$ & $33.9(10.2)$ & $+2.7(4.5) *$ & 1.0000 \\
\hline Time slope, sec $\cdot \mathrm{mL}^{-1} \cdot \min$ & $0.96(0.28)$ & $+0.07(0.30)$ & $0.98(0.34)$ & $+0.19(0.25)^{* *}$ & 0.3310 \\
\hline The causes to stop during CPET & Pre-PR & Post-PR & Pre-PR & Post-PR & Not done \\
\hline Dyspnea/leg fatigue & $14 / 6$ & $11 / 9$ & $11 / 7$ & $9 / 9$ & Not done \\
\hline
\end{tabular}

Data are presented as means (standard deviation). Peak $V_{E}^{\prime}$ : minute ventilation at peak exercise; inc.: increase PR: pulmonary rehabilitation; six-MWD: six-minute walking distance; CPET: cardiopulmonary exercise testing; $V_{\mathrm{O} 2}^{\prime}$ : oxygen uptake; R: gas exchange ratio; $V_{\mathrm{T}}$ : tidal volume; $f_{\mathrm{R}}$ : breathing frequency; $\mathrm{T}_{\mathrm{i}} / \mathrm{T}_{\text {tot }}$ : inspiratory duty cycle; $V_{\mathrm{D}} / V_{\mathrm{T}}$ : physiological dead space/tidal volume ratio; $\mathrm{HR}$ : heart rate; $\mathrm{O}_{2}$ pulse: $V_{\mathrm{O} 2}^{\prime} / \mathrm{HR} ; \mathrm{SpO}_{2}$ : percutaneous oxygen saturation; $\mathrm{Pet} \mathrm{CO}_{2}$ : partial pressure of end-tidal carbon dioxide; $\triangle \mathrm{FO}_{2}$ : difference between inspiratory and expiratory $\mathrm{O}_{2}$ concentrations; $V_{\mathrm{CO} 2}^{\prime}$ : carbon dioxide output; $V_{\mathrm{D}}$-intercept: $V_{\mathrm{D}}$ calculated as $V_{\mathrm{E}}^{\prime}$-axis intercept $/ f_{\mathrm{R}}$-axis intercept, obtained from $V_{\mathrm{E}}^{\prime} \mathrm{vs}$. $V_{\mathrm{CO} 2}^{\prime}$ and $f_{\mathrm{R}} \mathrm{vs} . V_{\mathrm{CO} 2}^{\prime}$ relationships (see the Methods section for details); $V_{\mathrm{E}}^{\prime} / V^{\prime} \mathrm{CO}_{2}$ nadir: the lowest value of the ratio between $V_{\mathrm{E}}^{\prime}$ and $V_{\mathrm{CO} 2}^{\prime}$ during exercise (see the Methods section for details); $V_{\mathrm{E}}^{\prime}-V^{\prime} \mathrm{CO}_{2}$ slope: the slope was determined by linear regression analysis of $V_{\mathrm{E}}^{\prime}$ to $V^{\prime} \mathrm{CO} 2$ obtained during exercise (see the Methods section for details); Time slope: ratio of exercise time until exhaustion to $\Delta V_{\mathrm{O} 2}^{\prime}$ (peak minus resting $V_{\mathrm{O} 2}^{\prime}$ ) during CPET (see the Methods section for details). ${ }^{*} p<0.05$, ${ }_{* *} p<0.01,{ }^{* * *} p<0.001$ : compared with pre-PR values (within-group difference). ${ }^{+}$The data of 6-MWD were not obtained from two patients. ${ }^{++}: 27$ patients (peak $V_{\mathrm{E}}^{\prime}$ inc. group, $n=15$; peak $V_{\mathrm{E}}^{\prime}$ non-increase group, $n=12$ ), whose anaerobic thresholds were obtained before and after pulmonary rehabilitation were analyzed. 
Peak $\Delta \mathrm{FO}_{2}$ inc. group $(\mathrm{n}=17)$

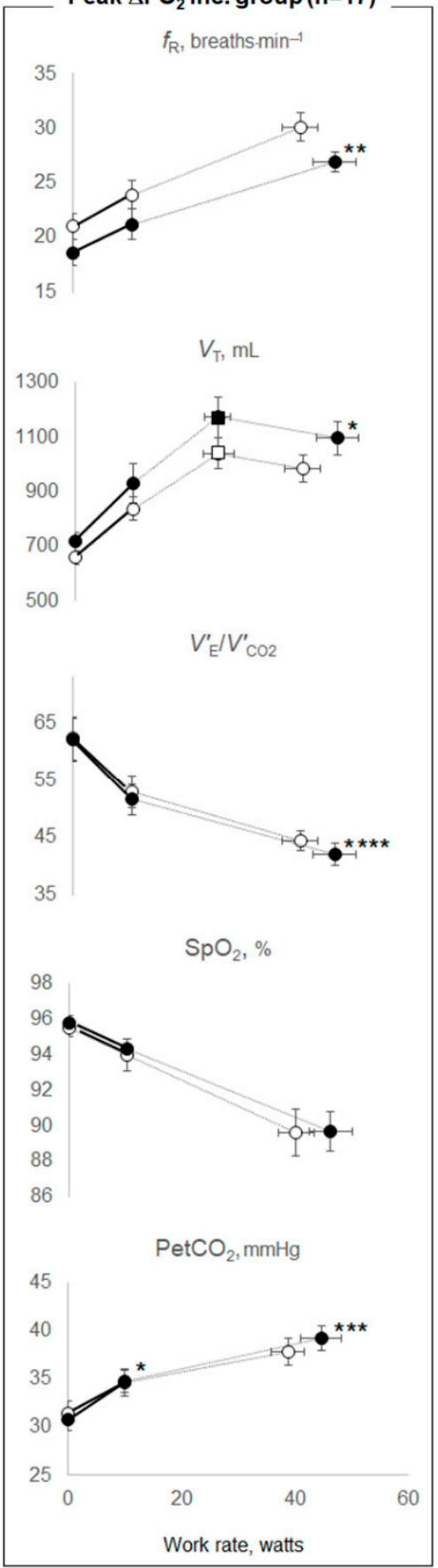

Peak $\Delta \mathrm{FO}_{2}$ non-inc. group $(\mathrm{n}=21)$

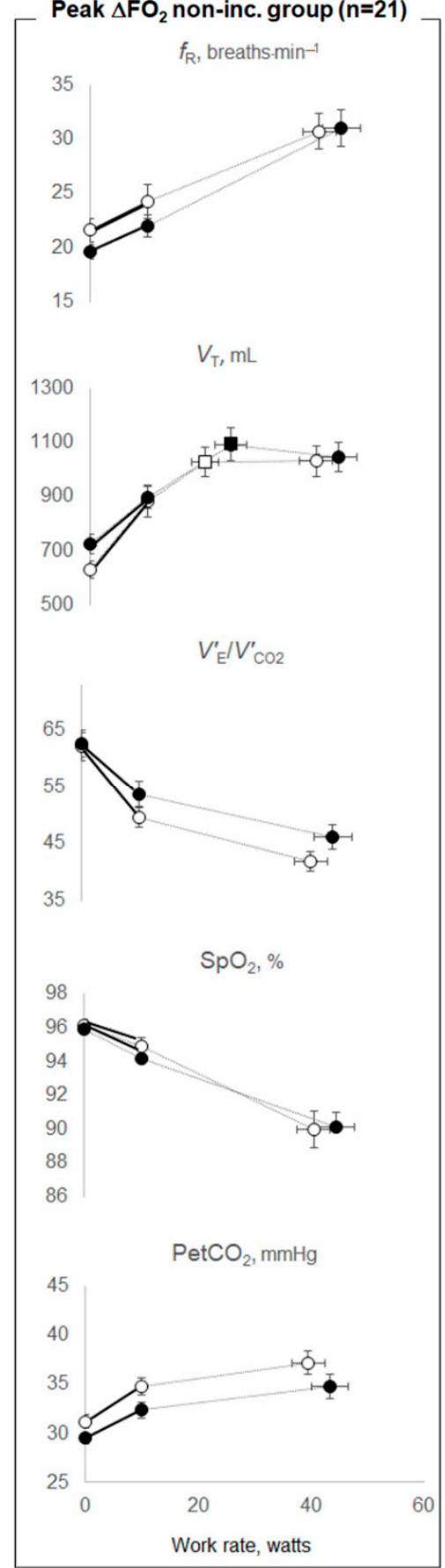

Figure 1. Exercise variables before and after pulmonary rehabilitation between the peak $\Delta \mathrm{FO}_{2}$ increase and non-increase groups. Data are presented as means (standard error). All patients were divided into two groups based on whether or not $\Delta \mathrm{FO}_{2}$ at peak exercise had increased after pulmonary rehabilitation. Peak $\Delta \mathrm{FO}_{2}$ : difference between inspiratory and expiratory $\mathrm{O}_{2}$ concentrations at peak exercise; inc.: increase; $f_{\mathrm{R}}$ : breathing frequency; $V_{\mathrm{T}}$ : tidal volume; $V_{\mathrm{E}}^{\prime}$ : minute ventilation; $V^{\prime}{ }_{\mathrm{CO}}$ : carbon dioxide output; $\mathrm{SpO}_{2}$ : percutaneous oxygen saturation; $\mathrm{PetCO}_{2}$ : partial pressure of end-tidal carbon dioxide. ${ }^{*} p<0.05,{ }^{* *} p<0.01,{ }^{* * *} p<0.001,{ }^{* * *} p<0.0001$ : mean differences between pre-and post-pulmonary rehabilitation values at each exercise phase were compared between peak $\Delta \mathrm{FO}_{2}$ increase and non-increase groups. Open symbols: before pulmonary rehabilitation. Closed symbols: after pulmonary rehabilitation. Squares represent $V_{\mathrm{T}}$-ventilation inflection points. 
Peak $V_{E}^{\prime}$ inc. group $(n=20)$

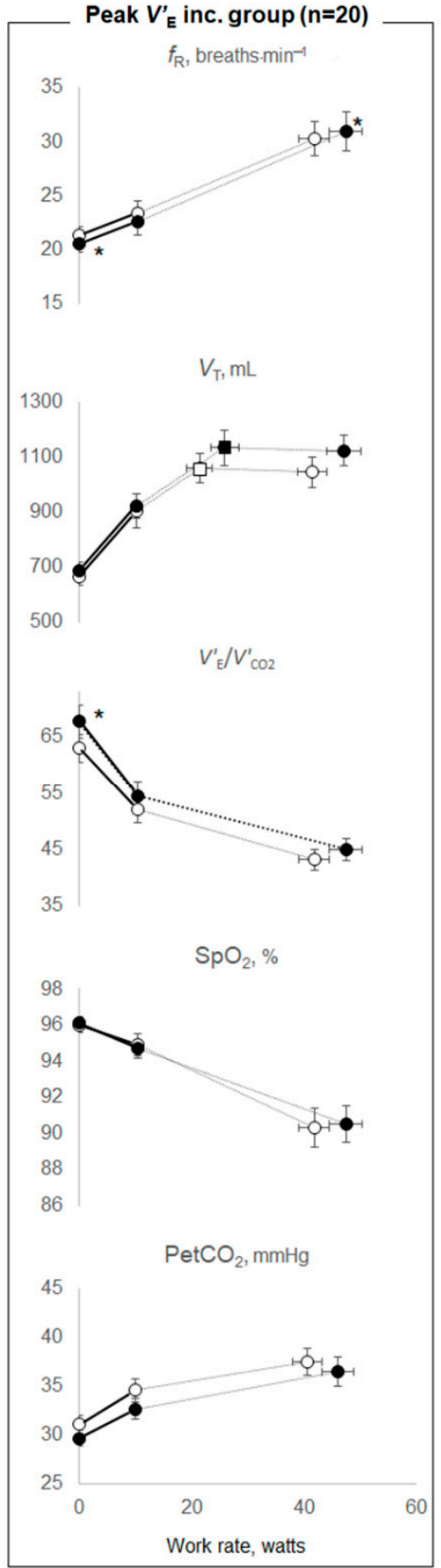

Peak $V_{E}^{\prime}$ non-inc. group $(\mathrm{n}=18)$

$f_{\mathrm{R}}$, breaths $\min ^{-1}$

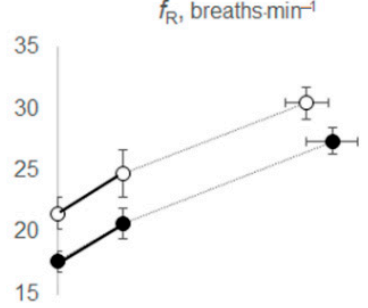

$\mathrm{V}_{\mathrm{T}}, \mathrm{mL}$

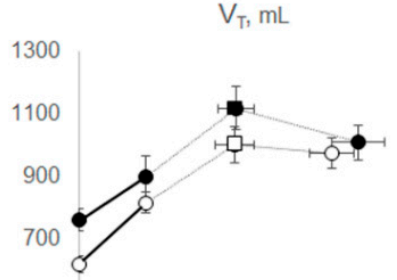

500

$V_{E}^{\prime} / V_{\mathrm{CO} 2}^{\prime}$

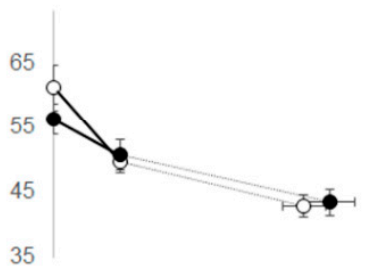

$\mathrm{SpO}_{2}, \%$

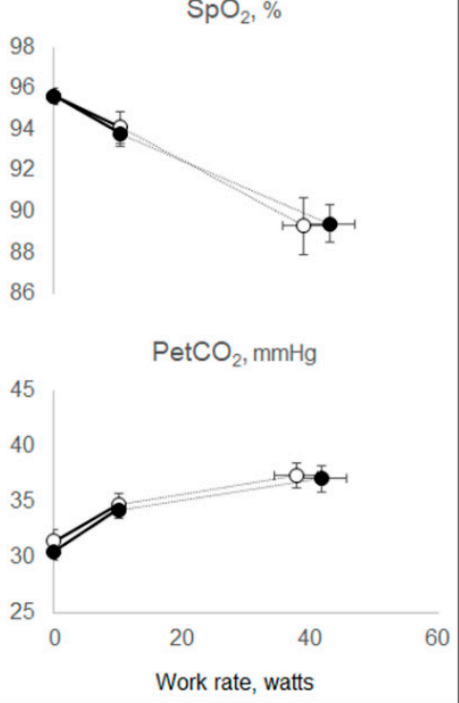

Figure 2. Exercise variables before and after pulmonary rehabilitation between the peak $V_{\mathrm{E}}^{\prime}$ increase and non-increase groups. Data are presented as means (standard error). All patients were divided into two groups based on whether or not $V_{\mathrm{E}}^{\prime}$ at peak exercise had increased after pulmonary rehabilitation. $V_{\mathrm{E}}^{\prime}$ : minute ventilation; inc.: increase; $f_{\mathrm{R}}$ : breathing frequency; $V_{\mathrm{T}}$ : tidal volume; $V_{\mathrm{CO} 2}^{\prime}$ : carbon dioxide output; $\mathrm{SpO}_{2}$ : percutaneous oxygen saturation; $\mathrm{PetCO}_{2}$ : partial pressure of end-tidal carbon dioxide. ${ }^{*} p<0.05$ : mean differences between pre-and post-pulmonary rehabilitation values at each exercise phase were compared between peak $V_{E}^{\prime}$ increase and non-increase groups. Open symbols: before pulmonary rehabilitation. Closed symbols: after pulmonary rehabilitation. Squares represent $V_{\mathrm{T}}$-ventilation inflection points. 
Next, we investigated whether the change in $\Delta \mathrm{FO}_{2}$ at peak exercise following PR correlated with changes in the other CPET variables, as shown in Table 6 and Figure 3. The difference in $\Delta \mathrm{FO}_{2}$ at peak exercise resulting from PR correlated positively with the difference following PR in peak $V^{\prime}{ }_{\mathrm{O} 2}(\mathrm{r}=0.4884, p=0.0019)$ and $V_{\mathrm{O} 2}^{\prime}$ at the anaerobic threshold $(\mathrm{r}=0.6711, p=0.0001)$ and correlated negatively with the difference following $\mathrm{PR}$ in $f_{\mathrm{R}}(\mathrm{r}=-0.3894, p=0.0157)$, the difference in $V_{\mathrm{E}}^{\prime} / V^{\prime} \mathrm{CO}_{2}$-nadir during exercise $(\mathrm{r}=-0.7057, p<0.0001)$ and the difference in $V^{\prime}{ }_{\mathrm{E}}-V^{\prime} \mathrm{CO}$-slope $(\mathrm{r}=-0.4578, p=0.0039)$. The change in $\mathrm{PetCO}_{2}$ at peak exercise following $\mathrm{PR}$ correlated with the difference in $V_{\mathrm{E}}^{\prime} / V^{\prime} \mathrm{CO}^{-}$ nadir during exercise $(\mathrm{r}=-0.5227, p<0.0001)$ and the difference in $V_{\mathrm{E}}^{\prime}-V_{\mathrm{CO}}^{\prime}$-slope $(\mathrm{r}=-0.3448, p=0.0340)$, although the significance of this was lower than the correlation with the change in $\Delta \mathrm{FO}_{2}$. In addition, the change in $\Delta \mathrm{FO}_{2}$ at peak exercise following PR was positively correlated with the time-slope before PR $(r=0.4120, p=0.0102)$. No significant correlation was confirmed between the change in $V^{\prime}{ }_{\mathrm{E}}$ at peak exercise and the change in $V_{\mathrm{E}}^{\prime} / V^{\prime} \mathrm{CO}$-nadir during exercise $(\mathrm{r}=0.0795, p=0.6352)$, and the change in $V_{\mathrm{E}}^{\prime}-V^{\prime} \mathrm{CO}^{-}$-slope ( $\mathrm{r}=0.0915, p=0.5850$ ) or between the change in $V_{\mathrm{E}}^{\prime}$ and the change in $\Delta \mathrm{FO}_{2}$ at peak exercise following PR $(\mathrm{r}=-0.0988, p=0.5552)$ (Table 6).

Table 6. Correlations between the change in the $\Delta \mathrm{FO}_{2}$ at peak exercise resulting from pulmonary rehabilitation and other parameters $(n=38)$.

\begin{tabular}{|c|c|c|}
\hline & $\mathbf{r}$ & $p$-Value \\
\hline Dyspnea at peak exercise diff., Borg scale & 0.1361 & 0.4153 \\
\hline Peak $V_{\mathrm{O} 2}^{\prime}$ diff., $\mathrm{mL} \cdot \mathrm{min}^{-1}$ & 0.4884 & 0.0019 \\
\hline$V_{\mathrm{O} 2}^{\prime}$ at anaerobic threshold diff., $\mathrm{mL} \cdot \mathrm{min}^{-1}$ & 0.6711 & 0.0001 \\
\hline$V_{\mathrm{E}}^{\prime}$ at peak exercise diff., $\mathrm{L} \cdot \mathrm{min}^{-1}$ & -0.0988 & 0.5552 \\
\hline$V_{\mathrm{T}}$ at peak exercise diff., $\mathrm{mL}$ & 0.2655 & 0.1072 \\
\hline$f_{\mathrm{R}}$ at peak exercise diff., breaths $\cdot \min ^{-1}$ & -0.3894 & 0.0157 \\
\hline$V_{\mathrm{D}} / V_{\mathrm{T}}$ at peak exercise diff. & -0.2428 & 0.1419 \\
\hline $\mathrm{O}_{2}$ pulse at peak exercise diff., $\mathrm{mL} \cdot$ beats $^{-1}$ & 0.2547 & 0.1228 \\
\hline$V_{\mathrm{E}}^{\prime} / V_{\mathrm{CO} 2}^{\prime}$-nadir diff. & -0.7057 & $<0.0001$ \\
\hline$V_{\mathrm{E}}^{\prime}-V_{\mathrm{CO} 2}^{\prime}$-slope diff. & -0.4578 & 0.0039 \\
\hline Time slope diff., $\mathrm{s} \cdot \mathrm{mL}^{-1} \cdot \min$ & -0.4518 & 0.0044 \\
\hline
\end{tabular}

$\mathrm{\Delta FO}_{2}$ : difference between inspiratory and expiratory $\mathrm{O}_{2}$ concentrations; Diff.: value after pulmonary rehabilitation minus the value before pulmonary rehabilitation; $V_{\mathrm{O} 2}^{\prime}$ : oxygen uptake; $V_{\mathrm{E}}^{\prime}$ : minute ventilation; $V_{\mathrm{T}}$ : tidal volume; $f_{\mathrm{R}}$ : breathing frequency; $V_{\mathrm{D}} / V_{\mathrm{T}}$ : physiological dead space/tidal volume ratio; $\mathrm{O}_{2}$ pulse: $V_{\mathrm{O} 2}^{\prime} /$ heart rate; $V^{\prime} \mathrm{CO}_{2}$ : carbon dioxide output; $V_{\mathrm{E}}^{\prime} / V^{\prime} \mathrm{CO}_{2}$-nadir: lowest value of the ratio between minute ventilation and carbon dioxide output during exercise (see the Methods section for details); $V^{\prime}{ }_{E}-V^{\prime} \mathrm{CO}_{2}$-slope: the slope was determined by linear regression analysis of minute ventilation to carbon dioxide output obtained during exercise (see the Methods section for details); Time slope: ratio of exercise time until exhaustion to obtained $\Delta V_{\mathrm{O} 2}^{\prime}$ (peak minus resting $V^{\prime} \mathrm{O} 2$ ) during cardiopulmonary exercise testing (see the Methods section for details). 


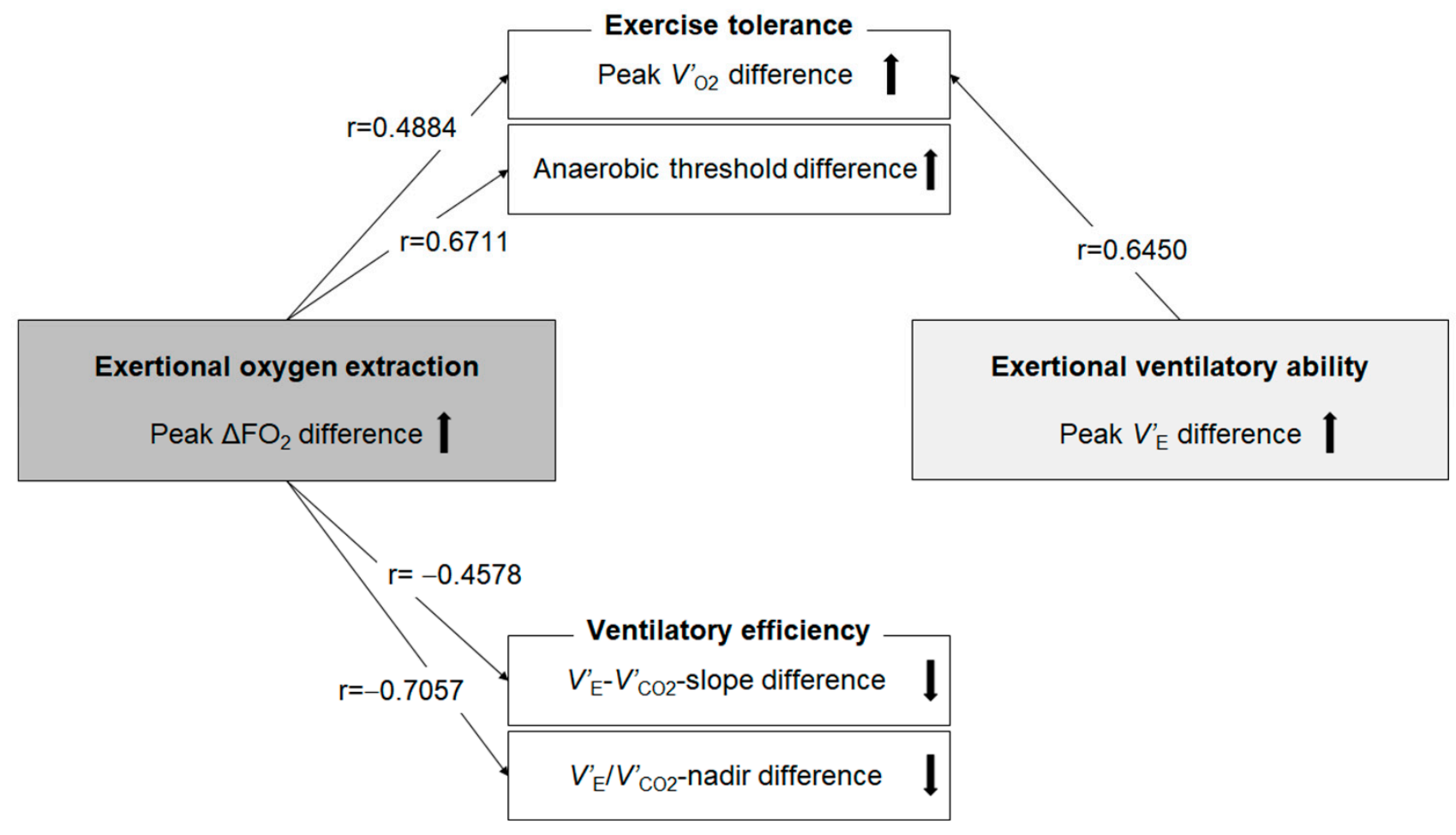

Figure 3. Correlations related to exercise tolerance and ventilatory efficiency. Difference: between before and after pulmonary rehabilitation; $\Delta \mathrm{FO}_{2}$ : difference between inspired and expired $\mathrm{O}_{2}$ concentration; $V_{\mathrm{E}}^{\prime}$ : minute ventilation; $V_{\mathrm{E}}^{\prime} / V_{\mathrm{CO} 2}^{\prime}$-nadir: lowest value of the ratio between minute ventilation and carbon dioxide output during exercise (see the Methods section for details); $V^{\prime}{ }^{-}$ $V^{\prime} \mathrm{CO}_{2}$-slope: the slope was determined by linear regression analysis of minute ventilation to carbon dioxide output observed during exercise (see the Methods section for details); $V_{\mathrm{O} 2}^{\prime}$ : oxygen uptake. Black arrows mean the up and down differences obtained from pulmonary rehabilitation.

\section{Discussion}

The present study aimed to investigate whether PR leads to an increase in incremental effort evaluated by CPET and whether the resultant change in $\mathrm{O}_{2}$ extraction affects the exertional pathophysiological conditions after PR in advanced COPD patients. The main observations were as follows. First, peak $V^{\prime}$ O2 was increased in only $14(37 \%)$ patients. Second, in the peak $\Delta \mathrm{FO}_{2}$ increase group (17 of 38 patients), exercise tolerance and ventilatory efficiency were improved by PR without an increase of $V_{E}^{\prime}$. In the peak $V_{\mathrm{E}}^{\prime}$ increase group (20 of 38 patients), although peak $V_{\mathrm{O} 2}^{\prime}$ was significantly increased, ventilatory efficiency did not improve. Third, in all the patients, the difference in $\mathrm{O}_{2}$ extraction at peak exercise before and after PR correlated positively with the difference in exercise tolerance, and negatively with the difference in ventilatory efficiency.

Increasing $\mathrm{O}_{2}$ extraction, which is evaluated as $\triangle \mathrm{FO}_{2}$ in CPET, would help improve exercise tolerance, including the anaerobic threshold and ventilatory efficiency, particularly in patients with advanced COPD (Figure 3). Based on the Fick principle, $V^{\prime}{ }_{2}$ is the product of cardiac output and the difference between arterial and mixed venous oxygen content. The difference in arteriovenous oxygen content reflects $\mathrm{O}_{2}$ extraction by the muscles [26]. In CPET, only ventilatory flow and the inspired and expired concentrations of $\mathrm{O}_{2}$ and $\mathrm{CO}_{2}$ are directly measured at the mouth; all other variables are calculated using these measurements. In CPET, $V^{\prime}{ }_{\mathrm{O} 2}$ is calculated using the product of $V_{\mathrm{E}}^{\prime}$ and $\Delta \mathrm{FO}_{2}[6,13]$. The latter reflects total $\mathrm{O}_{2}$ extraction related to cardiopulmonary-muscle crosstalk in the body. Of note, $\Delta \mathrm{FO}_{2}$ did not correlate with $V_{\mathrm{E}}^{\prime}$ at peak exercise in our previous study [19]. Furthermore, the change in $\Delta \mathrm{FO}_{2}$ at peak exercise following PR did not correlate with the change in $V_{\mathrm{E}}^{\prime}$ after PR in the present study (Table 6). These findings suggest that $\Delta \mathrm{FO}_{2}$ might be usable for CPET evaluations independent of $V^{\prime}$. However, little is known about 
whether this total $\mathrm{O}_{2}$ extraction affects exertional pathophysiological conditions including airflow limitation, cardiac dysfunction and metabolic changes in COPD patients. In the peak $\Delta \mathrm{FO}_{2}$ increase and non-increase groups, no significant treatment changes in $V_{\mathrm{T}}$ at the inflection point during exercise, in $\mathrm{Ti}$ / Ttot at peak exercise and in $\mathrm{O}_{2}$-pulse, i.e., the product of the stroke volume and arterial-venous $\mathrm{O}_{2}$ content difference, were observed (Table 4 and Figure 1); that is, the changed levels following PR of the mechanical constraints on $V_{\mathrm{T}}$ and the prolonged expiration pattern during exercise due to wasted ventilation and the changed levels of cardiac dysfunction were similar between the two groups. In addition, the difference in $\Delta \mathrm{FO}_{2}$ at peak exercise resulting from PR correlated with the difference following PR in peak $V^{\prime} \mathrm{O} 2$ and the anaerobic threshold (Table 6 and Figure 3), but it did not correlate with the difference following PR in $V_{E}^{\prime}$ or $\mathrm{O}_{2}$-pulse at peak exercise (Table 6). The response that the higher oxygen extraction obtained from PR improved exercise tolerance shifting the anaerobic threshold point to the late exercise phase might be caused by improved muscle condition related to $\mathrm{O}_{2}$ extraction rather than a direct cardiopulmonary mechanism in the cardiopulmonary-muscle crosstalk. Our previous report [19] and the present study demonstrated that $\Delta \mathrm{FO}_{2}$ at peak exercise and the change in $\Delta \mathrm{FO}_{2}$ following $\mathrm{PR}$, which is a gas exchange parameter related to $\mathrm{O}_{2}$, rather than $\mathrm{CO}_{2}-$ related variables, such as $\mathrm{PetCO}_{2}$, had a stronger inverse relationship with $V_{\mathrm{E}}^{\prime}-V_{\mathrm{CO}}^{\prime}$ slope and the change in the $V^{\prime}{ }_{\mathrm{E}}-V^{\prime} \mathrm{CO} 2$ slope following $\mathrm{PR}$, respectively. These findings suggest that the $V^{\prime}{ }_{E}-V^{\prime} \mathrm{CO}_{2}$ slope is a comprehensive variable that reflects not only $\mathrm{CO}_{2}$ gas exchangeability, but also $\mathrm{O}_{2}$ extraction ability. Not only that, they might illustrate the response, as shown in Figure 3, that higher oxygen extraction from PR had on improving ventilatory efficiency, as well as shifting the highest $V^{\prime}{ }_{\mathrm{O} 2}$ point without developing an exertional acidosis to the late exercise phase, and they were associating with each other. The response might be the reason why $f_{\mathrm{R}}$ at peak exercise was reduced after PR in the peak $\Delta \mathrm{FO}_{2}$ increase group (Table 4 ). In contrast, in the peak $V_{\mathrm{E}}^{\prime}$ increase group, peak $V_{\mathrm{O} 2}^{\prime}$ increased, but the increase in $V_{\mathrm{E}}^{\prime}$ after PR depended on tachypnea, and ventilatory efficiency was not improved after PR (Table 5 and Figure 2). Given the evidence that $V^{\prime} \mathrm{E}^{-}$ $V^{\prime} \mathrm{CO} 2$ slope is a prognostic factor in several chronic cardiopulmonary diseases independent of other exercise-related variables such as peak $V^{\prime}{ }_{2}[18]$, the present results suggest that much attention should be paid to the clinical information about $\mathrm{O}_{2}$ extraction. In terms of pre-PR parameters, only the time-slope in the peak $\Delta \mathrm{FO}_{2}$ increase group was higher than that in the peak $\Delta \mathrm{FO}_{2}$ non-increase group. In addition, the change in $\Delta \mathrm{FO}_{2}$ at peak exercise resulting from PR was positively correlated with the time-slope before PR. These findings indicate that the more gently patients exercised to obtain a certain $V_{\mathrm{O} 2}^{\prime}$ before PR the larger was the change in $\mathrm{O}_{2}$ extraction obtained after PR. Furthermore, in our previous study, the $\Delta \mathrm{FO}_{2}-V^{\prime} \mathrm{CO} 2$ slope during exercise correlated positively with the $\mathrm{PaCO}_{2}-V^{\prime} \mathrm{CO} 2$ slope, that is, the degree of exertional respiratory acidosis in COPD patients [19]. Although a slower rate of change in $V_{\mathrm{O} 2}^{\prime}$ for a given change in work rate is generally recognized in CPET responses, indicating poor $\mathrm{O}_{2}$ delivery or extraction [13], these findings suggest that increasing $\mathrm{O}_{2}$ extraction would not only improve exercise tolerance and ventilatory efficiency but might also play a compensatory or protective role during exercise in advanced COPD patients.

Improving exercise tolerance, especially incremental effort, following PR might be difficult in some patients, particularly in those with advanced COPD [11,12], although various strategies for improving exercise tolerance in COPD patients have been studied. Surprisingly, in the present study, PR resulted in a decrease in dyspnea at peak exercise even in the peak $\Delta \mathrm{FO}_{2}$ non-increase group, despite the lack of improvement in incremental effort. This could be explained by the assumption that less incremental effort required for exercise resulting from PR might have led to a decrease in dyspnea reducing $\mathrm{O}_{2}$ extraction because exercise tolerance and ventilatory efficiency could not improve sufficiently following PR. Meanwhile, given that international position statements recommend that PR programs should offer exercise training for 8-10 weeks, which is longer than the duration of PR in the present study [7], we speculate that a longer PR program might increase $\Delta \mathrm{FO}_{2}$, 
leading to improved exercise tolerance and hence might be needed in certain advanced COPD patients in whom an inability to improve incremental effort is expected. Though the investigation did not specifically address for treatment change in $\Delta \mathrm{FO}_{2}$ evaluated by CPET, we fortunately found that oxygen extraction was improved by the administration of ghrelin [27], which was first discovered to have a variety of effects including direct effects of vasodilation [28] and an increase in cardiac output [29]. We previously reported that activated ghrelin (acyl ghrelin) treatment without PR improved peak $V_{\mathrm{O} 2}^{\prime}$ in patients with severe and very severe COPD and that this effect might be attributed to the resultant improvements in cardiac function by $\mathrm{O}_{2}$ pulse and an increase in $\Delta \mathrm{FO}_{2}$ rather than $V_{\mathrm{E}}^{\prime}$ [30]. Thus, developing appropriate strategies for improving $\Delta \mathrm{FO}_{2}$, using the interaction of the pulmonary, cardiovascular and muscle crosstalk in the body, might lead to a clear understanding of the mechanism by which increasing $\mathrm{O}_{2}$ extraction improves exercise tolerance and gas exchange in COPD patients.

The present study has some limitations. First, this was a single-center study with a small number of patients, and the number of female patients was disproportionately low. Second, detailed evaluation of $\mathrm{O}_{2}$ delivery-utilization in skeletal muscles was not performed in the present study, although it has been reported that the skeletal muscle area measured by computed tomography correlates with ventilatory efficiency during CPET [31], and hence, would have correlated with $\mathrm{O}_{2}$ extraction during exercise in the present study and our previous study [19]. Third, the study included some COPD patients who regularly took short-acting muscarinic antagonists rather than long-acting muscarinic antagonists, and the number of patients receiving dual or triple therapy including inhalation therapy was low, which might have affected the results. Fourth, blood samples for blood gas analyses were not collected during CPET for the estimation of dead space values during exercise and elucidation of the pathophysiological mechanisms during exercise. Admittedly, two non-invasive estimations of dead space volume were used, but in both the peak $\Delta \mathrm{FO}_{2}$ or $V_{\mathrm{E}}^{\prime}$ increase and non-increase groups, no significant differences in the mean change following PR in $V_{\mathrm{D}} / V_{\mathrm{T}}$ estimated by Enghoff's modification of Bohr's equation and $V_{\mathrm{D}}$-intercept $/ V_{\mathrm{T}}$ were observed (Tables 4 and 5). Fifth, given that the optimal test duration for CPET should be from eight to twelve minutes [13,32], the relatively short test duration in the present study might have affected the results, especially for the calculations using exertional ventilatory variables vs. $V^{\prime} \mathrm{CO} 2$ relationship, such as the slope and $Y$ axis intercept. Sixth, no significant difference in improvement in exertional dyspnea at peak exercise was observed between the peak $\Delta \mathrm{FO}_{2}$ increase and non-increase groups. In addition, isotime comparisons showed that Borg scale scores tended to decrease after PR (mean Borg scale score, pre-PR $6.1 \pm 2.9$, post-PR $4.4 \pm 2.9$; within-group comparison, $p=0.0797$ ) in the peak $\Delta \mathrm{FO}_{2}$ increase group, indicating that the exertional dyspnea was reduced at isotime. However, the change was not significantly different between the peak $\Delta \mathrm{FO}_{2}$ increase and non-increase groups $(p=0.6177)$. The resultant endurance effort rather than the incremental effort obtained from PR might affect the decrease in exertional dyspnea in the peak $\Delta \mathrm{FO}_{2}$ non-increase group, although it may be difficult to distinguish the effect of endurance effort if an incremental effort was not obtained from PR. Further studies including a specific treatment strategy targeting oxygen extraction might be necessary to confirm whether increasing oxygen extraction is useful for improving exertional dyspnea.

\section{Conclusions}

PR resulted in increased exercise tolerance in only $37 \%$ of patients with advanced COPD in the present study. The change in $\mathrm{O}_{2}$ extraction ability resulting from $\mathrm{PR}$, evaluated as the $\Delta \mathrm{FO}_{2}$ during exercise, correlated positively with the post-PR change in exercise tolerance and the anaerobic threshold, and negatively with the change in ventilatory efficiency. In patients with advanced COPD, it is often difficult to increase ventilatory ability because of the dynamically hyperinflated lungs. Hence, new strategies involving the improvement of $\mathrm{O}_{2}$ extraction ability are needed for better exercise tolerance and improved ventilatory efficiency. 
Author Contributions: All authors contributed substantially to this article. A.M. and K.M. were responsible for the study conception and design. A.M., K.M., R.M., K.T., H.H., H.Y., M.M., T.K., T.N., T.M. and H.K. were responsible for data acquisition, analysis and interpretation. A.M., K.M., R.M. K.T., H.H., H.Y., M.M., T.K., T.N., T.M. and H.K. were responsible for drafting and revising the article. All authors have read and agreed to the published version of the manuscript.

Funding: This work was supported by a Grant-in-Aid for Clinical Research from the National Hospital Organization (number, not applicable). The funder had no role in the study design, data collection and analysis, or preparation of the manuscript.

Institutional Review Board Statement: The institutional review board of the National Hospital Organization Osaka Toneyama Medical Center approved the study protocol (approval number: TNH-A-2021022) and the protocol was in accordance with the Declaration of Helsinki for experiments involving human subjects.

Informed Consent Statement: The patients/participants provided their written informed consent to participate in this study.

Data Availability Statement: The data that support the findings of this study are available from the corresponding author upon reasonable request.

Acknowledgments: The authors would like to thank S. Sakaguchi, S. Ito, and K. Koyama for their help with the CPET measurements.

Conflicts of Interest: The authors declare that they have no conflict of interest.

\section{Abbreviations}

CPET: cardiopulmonary exercise testing; $\Delta \mathrm{FO}_{2}$, difference between inspiratory and expiratory oxygen concentrations; GOLD, Global Initiative for Chronic Obstructive Lung Disease; SAMA, short-acting muscarinic antagonist; LAMA, long-acting muscarinic antagonist; SABA, short-acting $\beta 2$-agonist; LABA, long-acting $\beta 2$-agonist; ICS, inhaled corticosteroid.

\section{References}

1. World Health Organization. The Top 10 Causes of Death. Available online: https://www.who.int/news-room/fact-sheets/ detail/the-top-10-causes-of-death (accessed on 15 October 2021).

2. Laviolette, L.; Laveneziana, P. Dyspnoea: A multidimensional and multidisciplinary approach. Eur. Respir. J. 2014, 43, 1750-1762. [CrossRef]

3. Miki, K. Motor Pathophysiology Related to Dyspnea in COPD Evaluated by Cardiopulmonary Exercise Testing. Diagnostics 2021, 11, 364. [CrossRef] [PubMed]

4. $\quad$ O'Donnell, D.E.; Milne, K.M.; James, M.D.; de Torres, J.P.; Neder, J.A. Dyspnea in COPD: New Mechanistic Insights and Management Implications. Adv. Ther. 2020, 37, 41-60. [CrossRef]

5. Riley, C.M.; Sciurba, F.C. Diagnosis and Outpatient Management of Chronic Obstructive Pulmonary Disease: A Review. JAMA 2019, 321, 786-797. [CrossRef] [PubMed]

6. Laviolette, L.; Laveneziana, P. Exercise Testing in the prognostic evaluation of patients with lung and heart diseases. In Clinical Exercise Testing (ERS Monograph); Palange, P., Laveneziana, P., Neder, J.A., Ward, S.A., Eds.; European Respiratory Society: Sheffield, UK, 2018; pp. 222-234.

7. Garvey, C.; Bayles, M.P.; Hamm, L.F.; Hill, K.; Holland, A.; Limberg, T.M.; Spruit, M.A. Pulmonary Rehabilitation Exercise Prescription in Chronic Obstructive Pulmonary Disease: Review of Selected Guidelines: An Official Statement from the American Association of Cardiovascular and Pulmonary Rehabilitation. J. Cardiopulm. Rehabil. Prev. 2016, 36, 75-83. [CrossRef]

8. Spruit, M.A.; Singh, S.J.; Garvey, C.; ZuWallack, R.; Nici, L.; Rochester, C.; Hill, K.; Holland, A.E.; Lareau, S.C.; Man, W.D.; et al. An official American Thoracic Society/European Respiratory Society statement: Key concepts and advances in pulmonary rehabilitation. Am. J. Respir. Crit. Care Med. 2013, 188, e13-e64. [CrossRef] [PubMed]

9. Camillo, C.A.; Langer, D.; Osadnik, C.R.; Pancini, L.; Demeyer, H.; Burtin, C.; Gosselink, R.; Decramer, M.; Janssens, W.; Troosters, T. Survival after pulmonary rehabilitation in patients with COPD: Impact of functional exercise capacity and its changes. Int. J. Chronic Obstr. Pulm. Dis. 2016, 11, 2671-2679. [CrossRef]

10. Maekura, R.; Hiraga, T.; Miki, K.; Kitada, S.; Miki, M.; Yoshimura, K.; Yamamoto, H.; Kawabe, T.; Mori, M. Personalized pulmonary rehabilitation and occupational therapy based on cardiopulmonary exercise testing for patients with advanced chronic obstructive pulmonary disease. Int. J. Chronic Obstr. Pulm. Dis. 2015, 10, 1787-1800. [CrossRef] 
11. Burtin, C.; Saey, D.; Saglam, M.; Langer, D.; Gosselink, R.; Janssens, W.; Decramer, M.; Maltais, F.; Troosters, T. Effectiveness of exercise training in patients with COPD: The role of muscle fatigue. Eur. Respir. J. 2012, 40, 338-344. [CrossRef]

12. Miki, K.; Maekura, R.; Kitada, S.; Miki, M.; Yoshimura, K.; Yamamoto, H.; Kawabe, T.; Kagawa, H.; Oshitani, Y.; Satomi, A.; et al Pulmonary rehabilitation for COPD improves exercise time rather than exercise tolerance: Effects and mechanisms. Int. J. Chronic Obstr. Pulm. Dis. 2017, 12, 1061-1070. [CrossRef]

13. Wasserman, K.; Hansen, J.; Sue, D.; Stringer, W.; Sietsema, K.; Sun, X.-G. Principles of Exercise Testing and Interpretation: Including Pathophysiology and Clinical Applications, 5th, ed.; Lippincott Williams and Wilkins: Philadelphia, PA, USA, 2012.

14. Oga, T.; Nishimura, K.; Tsukino, M.; Sato, S.; Hajiro, T. Analysis of the factors related to mortality in chronic obstructive pulmonary disease: Role of exercise capacity and health status. Am. J. Respir. Crit. Care Med. 2003, 167, 544-549. [CrossRef]

15. Yoshimura, K.; Maekura, R.; Hiraga, T.; Miki, K.; Kitada, S.; Miki, M.; Tateishi, Y.; Mori, M. Identification of three exercise-induced mortality risk factors in patients with COPD. COPD J. Chronic Obstr. Pulm. Dis. 2014, 11, 615-626. [CrossRef]

16. Neder, J.A.; Berton, D.C.; Arbex, F.F.; Alencar, M.C.; Rocha, A.; Sperandio, P.A.; Palange, P.; O’Donnell, D.E. Physiological and clinical relevance of exercise ventilatory efficiency in COPD. Eur. Respir. J. 2017, 49, 1602036. [CrossRef] [PubMed]

17. Phillips, D.B.; Collins, S.; Stickland, M.K. Measurement and Interpretation of Exercise Ventilatory Efficiency. Front. Physiol. 2020, 11, 659. [CrossRef]

18. Weatherald, J.; Sattler, C.; Garcia, G.; Laveneziana, P. Ventilatory response to exercise in cardiopulmonary disease: The role of chemosensitivity and dead space. Eur. Respir. J. 2018, 51, 1700860. [CrossRef] [PubMed]

19. Miki, K.; Tsujino, K.; Maekuara, R.; Matsuki, T.; Miki, M.; Hashimoto, H.; Kagawa, H.; Kawasaki, T.; Kuge, T.; Kida, H. Oxygen Extraction Based on Inspiratory and Expiratory Gas Analysis Identifies Ventilatory Inefficiency in Chronic Obstructive Pulmonary Disease. Front. Physiol. 2021, 12, 703977. [CrossRef] [PubMed]

20. GOLD. Global Strategy for the Diagnosis, Management, and Prevention of Chronic Obstructive Pulmonary Disease (2020 Report). Available online: https://goldcopd.org/wp-content/uploads/2019/12/GOLD-2020-FINAL-ver1.2-03Dec19_WMV.pdf (accessed on 15 October 2021).

21. Miki, K.; Maekura, R.; Nagaya, N.; Nakazato, M.; Kimura, H.; Murakami, S.; Ohnishi, S.; Hiraga, T.; Miki, M.; Kitada, S.; et al Ghrelin treatment of cachectic patients with chronic obstructive pulmonary disease: A multicenter, randomized, double-blind, placebo-controlled trial. PLoS ONE 2012, 7, e35708. [CrossRef] [PubMed]

22. Crapo, R.O.; Hankinson, J.L.; Irvin, C.; MacIntyre, N.R.; Voter, K.Z.; Wise, R.A.; Graham, B.; O’Donnell, C.; Paoletti, P.; Roca, J.; et al. Standardization of Spirometry: 1994 Update. Am. J. Respir. Crit. Care Med. 1995, 152, 1107-1136. [CrossRef]

23. Woo, M.A.; Moser, D.K.; Stevenson, L.W.; Stevenson, W.G. Six-minute walk test and heart rate variability: Lack of association in advanced stages of heart failure. Am. J. Crit. Care 1997, 6, 348-354. [CrossRef]

24. Gargiulo, P.; Apostolo, A.; Perrone-Filardi, P.; Sciomer, S.; Palange, P.; Agostoni, P. A non invasive estimate of dead space ventilation from exercise measurements. PLoS ONE 2014, 9, e87395. [CrossRef]

25. Hey, E.N.; Lloyd, B.B.; Cunningham, D.J.; Jukes, M.G.; Bolton, D.P. Effects of various respiratory stimuli on the depth and frequency of breathing in man. Respir. Physiol. 1966, 1, 193-205. [CrossRef]

26. Whipp, B.J.; Ward, S.A. Cardiopulmonary coupling during exercise. J. Exp. Biol. 1982, 100, 175-193. [CrossRef]

27. Kojima, M.; Hosoda, H.; Date, Y.; Nakazato, M.; Matsuo, H.; Kangawa, K. Ghrelin is a growth-hormone-releasing acylated peptide from stomach. Nature 1999, 402, 656-660. [CrossRef]

28. Okumura, H.; Nagaya, N.; Enomoto, M.; Nakagawa, E.; Oya, H.; Kangawa, K. Vasodilatory effect of ghrelin, an endogenous peptide from the stomach. J. Cardiovasc. Pharmacol. 2002, 39, 779-783. [CrossRef] [PubMed]

29. Nagaya, N.; Uematsu, M.; Kojima, M.; Ikeda, Y.; Yoshihara, F.; Shimizu, W.; Hosoda, H.; Hirota, Y.; Ishida, H.; Mori, H.; et al Chronic administration of ghrelin improves left ventricular dysfunction and attenuates development of cardiac cachexia in rats with heart failure. Circulation 2001, 104, 1430-1435. [CrossRef] [PubMed]

30. Miki, K.; Kitada, S.; Miki, M.; Hui, S.P.; Shrestha, R.; Yoshimura, K.; Tsujino, K.; Kagawa, H.; Oshitani, Y.; Kida, H.; et al. A phase II, open-label clinical trial on the combination therapy with medium-chain triglycerides and ghrelin in patients with chronic obstructive pulmonary disease. J. Physiol. Sci. 2019, 69, 969-979. [CrossRef] [PubMed]

31. Tashiro, H.; Takahashi, K.; Tanaka, M.; Sadamatsu, H.; Kurihara, Y.; Tajiri, R.; Takamori, A.; Naotsuka, H.; Imaizumi, H.; Kimura, S.; et al. Skeletal muscle is associated with exercise tolerance evaluated by cardiopulmonary exercise testing in Japanese patients with chronic obstructive pulmonary disease. Sci. Rep. 2021, 11, 15862. [CrossRef]

32. Buchfuhrer, M.J.; Hansen, J.E.; Robinson, T.E.; Sue, D.Y.; Wasserman, K.; Whipp, B.J. Optimizing the exercise protocol for cardiopulmonary assessment. J. Appl. Physiol. 1983, 55, 1558-1564. [CrossRef] 